Preprint typeset in JHEP style - HYPER VERSION

MIT-CTP-3611

hep-th/0503177

\title{
A New Infinite Class of Quiver Gauge Theories
}

\author{
Amihay Hanany, Pavlos Kazakopoulos, Brian Wecht \\ Center for Theoretical Physics, Massachusetts Institute of Technology, \\ Cambridge, MA 02139, USA. \\ hanany, noablake, bwecht@mit.edu
}

\begin{abstract}
We construct a new infinite family of $\mathcal{N}=1$ quiver gauge theories which can be Higgsed to the $Y^{p, q}$ quiver gauge theories. The dual geometries are toric Calabi-Yau cones for which we give the toric data. We also discuss the action of Seiberg duality on these quivers, and explore the different Seiberg dual theories. We describe the relationship of these theories to five dimensional gauge theories on $(\mathrm{p}, \mathrm{q})$ 5-branes. Using the toric data, we specify some of the properties of the corresponding dual Sasaki-Einstein manifolds. These theories generically have algebraic R-charges which are not quadratic irrational numbers. The metrics for these manifolds still remain unknown.
\end{abstract}

KeYwords: Quiver Gauge Theories, Five Dimensional Gauge Theories. 


\section{Contents}

1. Introduction 1

2. Warm-Ups: del Pezzo 2 and the Suspended Pinch Point 4

3. New quiver theories: $X^{p, q}$ 8

3.1 Review of the $Y^{p, q}$ quivers 9

$3.2 X^{p, q}$ for $1 \leq q \leq p-1$

$3.3 X^{p, q}$ for $q=p$

3.4 An example: $X^{3,1}, X^{3,2}$, and $X^{3,3} 13$

3.5 Toric Diagrams for $X^{p, q}$

田. (p,q)-brane webs and 5d gauge theories 17

5. Toric Phases of the $X^{p, q}$ theories 21

6. R-charges 25

7. Acknowledgements 28

\section{Introduction}

The past few months have brought exciting new developments in the study of $\mathcal{N}=1$ superconformal field theories. It is known that such field theories can be constructed in string theory by placing a stack of D3-branes at the tip of a non-compact Calabi-Yau cone; the first such examples of these theories were given for Calabi-Yau spaces which are orbifolds of $\mathbb{C}^{3}$ [1]. More generally, the AdS/CFT correspondence [2, 3, 他 says that there is a gauge/string duality which can be seen by taking the near horizon limit of the D3-branes. The simplest example, where the branes are placed in flat ten dimensional space, gives a duality between type IIB string theory on $A d S_{5} \times S^{5}$ and $\mathcal{N}=4$ SUSY Yang-Mills. More generally, however, we can place the D3-branes at the tip of a Calabi-Yau cone whose base is a five-dimensional Einstein-Sasaki manifold $X_{5}$; the near-horizon limit of this is then Type IIB on $A d S_{5} \times X_{5}$ [5, 6]. This setup breaks additional supersymmetry, generically leaving an $\mathcal{N}=1$ theory on the D3-branes. These $\mathcal{N}=1$ theories are quiver gauge theories [1], i.e. theories with product gauge groups and matter transforming in bifundamental representations. 
Ideally, one would like to know the metric on the Calabi-Yau manifold, since this would provide a great deal of information (e.g. volumes of calibrated submanifolds) which could be translated into data about the field theory. In practice, however, it is a difficult task to find these metrics, and until recently the metrics on only two Einstein-Sasaki five-manifolds were known: $S^{5}$ and $T^{1,1}$. Recently, an infinite family of Einstein-Sasaki manifolds with topology $S^{2} \times S^{3}$ were found by Gauntlett, Sparks, Martelli and Waldram [7]; these extend previous work by these authors on compactifications of M-theory [8]. The new Einstein-Sasaki spaces have been dubbed $Y^{p, q}$, where $p$ and $q$ are integers with $0<q<p$; the Calabi-Yau cones over these spaces are toric. Sparks and Martelli [9] recently computed the corresponding toric diagrams and found that $Y^{2,1}$ was in fact the horizon of the complex cone over the first del Pezzo surface $d P_{1}$. The corresponding gauge theory is known, having been computed via partial resolution of the orbifold $\mathbb{C}^{3} / \mathbb{Z}_{3} \times \mathbb{Z}_{3}[10]$. This begged the question: What are the gauge theories dual to the other $Y^{p, q}$ ?

The problem of finding these gauge theories was quickly solved. The authors of [11] constructed the dual $\mathcal{N}=1$ gauge theories (also called $Y^{p, q}$ ), thus providing an infinite family of AdS/CFT duals. The $Y^{p, q}$ theories have gauge group $S U(N)^{2 p}$, and the complete matter content and superpotentials are known. These theories have survived many nontrivial checks of the AdS/CFT duality, such as matching volumes of 3-manifolds in the Calabi-Yau with dimensions of operators in the gauge theory computed via $a$-maximization [12]. The $a$-maximization calculation was done for $Y^{2,1}$ in [13], and the authors of [11] found a general formula for the R-charges for any $Y^{p, q}$; these agree beautifully with the corresponding volume calculations in the string dual. For interesting related work on these $Y^{p, q}$ theories, see [14, 15, 16, 17, 18, 19, 20].

Some of the $Y^{p, q}$ theories and geometries are already familiar. We can formally extend the range of $q$ to $0 \leq q \leq p$ and find that $Y^{p, p}=S^{5} / \mathbb{Z}_{2 p}$, and $Y^{p, 0}=T^{1,1} / \mathbb{Z}_{p}$. These spaces are not smooth, but can be used as interesting string backgrounds. The corresponding gauge theories to these spaces are well-known [1, 10, 21, 22], and the rest of the $Y^{p, q}$ gauge theories were constructed by a simple procedure that generalizes the method for going from $Y^{2,2}=S^{5} / \mathbb{Z}_{2} \times \mathbb{Z}_{2}$ to $Y^{2,1}=d P_{1}$ to $Y^{2,0}=\mathbf{F}_{0}$.

The fact that the $Y^{p, q}$ spaces are toric is crucial to the above calculations. The toric diagram for a given geometry can be described as points in a three-dimensional lattice; these points describe a $\mathbb{C}^{*}$ action and uniquely specify the resulting geometry. In this sense, toric geometries are generalizations of $\mathbb{C P}^{n}$. Equivalently, we can describe the geometry with a toric fan, which is a series of vectors ending on the specified lattice points. A toric Calabi-Yau cone then satisfies the additional restriction that the endpoints of these vectors lie in a plane. For this reason, all the toric diagrams we study can be represented on a two-dimensional lattice. For good reviews of toric geometry, see 23, 24, 25].

The construction of quiver gauge theories from toric singularities is a difficult task and many attempts at getting a general formula have been made so far with some or partial success. The 
general problem is to start with a geometric description, given by the toric data, and then to use it in order to find the corresponding gauge theory that lives on a D3 brane probing the corresponding Calabi-Yau cone. There are a few steps in finding the quiver gauge theory: First, one looks for the gauge groups. The second step is to determine the matter bifundamental fields, and finally one must find the corresponding superpotential which encodes the interaction terms. We have described these three steps in order by level of difficulty; the third step is often really a hard problem to solve. If for some reason in an independent computation we have a good guess for the quiver gauge theory then it is a straightforward task to compute the corresponding toric data [10]. However, the current known techniques are very long in computation time and are practical only for relatively small (few gauge groups and few matter fields) quiver gauge theories.

Until the computation of the $Y^{p, q}$ quiver gauge theories there were only finitely many toric singularities with known quiver gauge theories, most of which were centered around del Pezzo surfaces and toric diagrams with one or no internal points. This situation changed when the $Y^{p, q}$ 's were found. This leads to the hope that there are many more such infinite families of quiver gauge theories for which the computation is a relatively simple task. This is going to be the point of this paper - to introduce another infinite family of quiver gauge theories for which we know the toric data.

Shortly after the computation of the $Y^{p, q}$ quivers it was realized that the toric diagrams had actually been studied before in a seemingly unrelated environment. In [25, 26] the $Y^{p, q}$ toric diagrams were shown to be dual to a 5-brane web which describes the dynamics of a five dimensional SYM $S U(p)$ gauge theory with 8 supercharges, with $q$ denoting the coefficient of a Chern Simons term. Only a few computations have been made since then [27, 28] and many more may be found. Using the connection to five dimensional gauge theories, we are able to borrow ideas from the construction of such theories using branes. In particular the process of adding flavors to the five dimensional gauge theory is a useful tool. As we will see this will be interpreted as an inverse Higgs mechanism (unhiggsing) for the quiver gauge theories and essentially is the main tool which allows the construction of the new infinite family of quiver theories. The correspondence with five dimensional theories thus turns out to be useful and may be used in future attempts to find other quiver theories.

In this paper, we describe a new infinite class of toric geometries and the corresponding gauge theories. To motivate this construction, consider the case of the (complex cone over the) second del Pezzo surface $d P_{2}$. Since $d P_{2}$ is simply $\mathbb{P}^{2}$ blown up at two points, it is easy to see that one can blow down an exceptional $\mathbb{P}^{1}$ to arrive at $d P_{1}$. This fact is reflected in the corresponding gauge theories via Higgsing of the $d P_{2}$ theory to the $d P_{1}$ theory by giving an appropriate bifundamental field a vacuum expectation value. Additionally, however, one can also blow down $d P_{2}$ to $\mathbb{F}_{0}$. As with $d P_{1}$, this can also be seen from the gauge theory via Higgsing. Thus, $d P_{2}$ is an example of a theory which can be Higgsed to give two different $Y^{p, q}$ theories. The goal of the present paper is to 
generalize this construction. The analogous phenomenon in the context of five dimensional gauge theories with $S U(2)$ gauge group was studied in [25, 26, 29, where it is associated with a discrete $\theta$ angle and the limit of a gauge theory with one flavor that has a large mass. The sign of the mass then implies which of the resulting theories is either $\mathbf{F}_{0}$ or $d P_{1}$.

We find a general procedure for constructing four dimensional $\mathcal{N}=1 S U(N)^{2 p+1}$ gauge theories we denote as $X^{p, q}$, which Higgs to $Y^{p, q}$ and $Y^{p, q-1}$. We present the toric diagrams, quivers, and superpotentials for these theories. The Higgsing process can be seen in a number of ways; the toric diagrams, quiver, and (p,q) 5-brane webs all provide illuminating perspectives, which we describe in detail. In addition, we discuss the different toric phases of the $X^{p, q}$ theories, i.e. the quivers one can get by Seiberg duality that are still $S U(N)^{2 p+1}$ gauge theories.

The outline of this paper is as follows. In Section 2, we motivate the construction of the $X^{p, q}$ theories by doing two simple examples. We first explore the relationship between $d P_{2}, d P_{1}$, and $\mathbb{F}_{0}$ from both the gauge theory and the toric perspectives. In addition, we describe an analogous relationship between the Suspended Pinch Point (SPP), conifold, and $\mathbb{C}^{3} / \mathbb{Z}_{2}$. In Section 3 , we describe the construction of one phase of the $X^{p, q}$ spaces. It is necessary to treat two cases, $q=p$ and $1 \leq q \leq p-1$, which we do. As a detailed example, we write down the quivers and superpotentials for the $X^{3,1}, X^{3,2}$, and $X^{3,3}$ theories. Finally, we discuss the toric diagrams for the dual $X^{p, q}$ geometries. In Section 4, we review the relationship of toric geometry with webs of $(\mathrm{p}, \mathrm{q})$ 5-branes, and describe how the Higgsing process can be seen from this perspective. We discuss how many parameters of the four dimensional theory are related to parameters of the five dimensional theory. In Section 5, we discuss the Seiberg dual phases of the $X^{p, q}$ theories that still

have gauge group $S U(N)^{2 p+1}$; these are usually called the "toric phases" of the theory. There is a general relationship between the number of bifundamentals in the different toric phases of the $X^{p, q}$ with the number of bifundamentals in the different toric phases of $Y^{p, q}$ and $Y^{p, q-1}$, which we discuss. Finally, in section 6 , we discuss the R-charges for the $X^{p, q}$ theories. The calculation is in general computationally quite difficult but can be done for some small values of $p$. We calculate the R-charges for $X^{2,2}$ and $X^{3,3}$ and find that they not quadratic irrational but instead the roots of quartic equations. In addition, we discuss how although $X^{2,1}$ has a two-dimensional basis of R-charges, this property does not appear to be true for larger values of $p$. This remains puzzling in light of recent results 30 .

\section{Warm-Ups: del Pezzo 2 and the Suspended Pinch Point}

Before we proceed to the general construction, we review the case of the gauge theory dual to the cone over $d P_{2}$. This theory provides a template for constructing the more general quivers, and as such is a useful example to explore.

Although we do not know the explicit metric on $d P_{2}$, the gauge theory has been constructed by partially resolving the space $\mathbb{C}^{3} / \mathbb{Z}_{3} \times \mathbb{Z}_{3}[10,22$. One quiver for this theory is given in Figure 


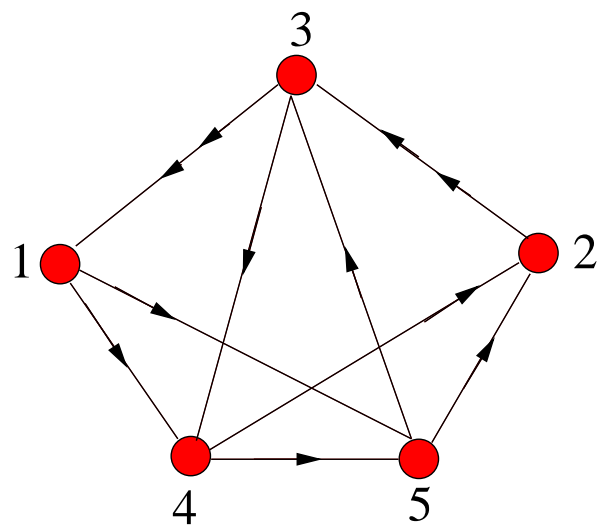

Figure 1: One quiver for the $d P_{2}$ gauge theory.

The accompanying superpotential is given by

$$
\begin{aligned}
W & =X_{34} X_{45} X_{53}-X_{53} Y_{31} X_{15}-X_{34} X_{42} Y_{23} \\
& +Y_{23} X_{31} X_{15} X_{52}+X_{42} X_{23} Y_{31} X_{14}-X_{23} X_{31} X_{14} X_{45} X_{52}
\end{aligned}
$$

This superpotential has 3 cubic terms, 2 quartics, and one quintic.

For our purposes in this work, the interesting thing to notice about the $d P_{2}$ quiver is that it can be Higgsed to either $Y^{2,1}$ or $Y^{2,0}$ by giving a vev to (for example) $X_{52}$ or $X_{45}$, respectively. See Figure 2 for these quivers. The Higgsing is straightforward: Giving a vev to a bifundamental field simply breaks the $S U(N) \times S U(N)$ group under which it transforms to the diagonal. This means that we should combine those two nodes in the quiver and delete the bifundamental from the theory this flows to in the IR. If there is a cubic term with the bifundamental in it, then we should also delete the other bifundamental fields; the vev will give them a mass and they should be integrated out of the IR theory.
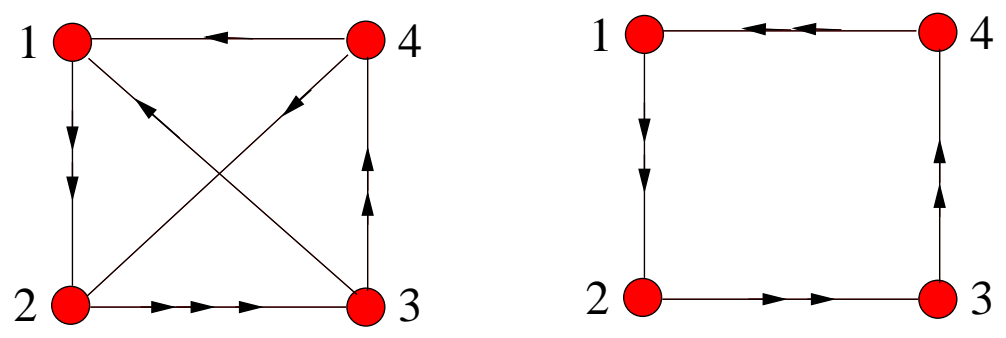

Figure 2: The quivers for $Y^{2,1}$ (left) and $Y^{2,0}$ (right)

To be complete, we must also check that the superpotential behaves as it should under Higgsing. Giving a vev to $X_{52}$ is straightforward: Since this bifundamental only appears in terms with four or 
more fields, we simply delete it from the appropriate terms and relabel the remaining fields. This yields the superpotential

$$
\begin{aligned}
W_{d P_{1}} & =X_{31} Y_{12} X_{23}-X_{23} Y_{34} X_{42}-X_{31} X_{12} Y_{23} \\
& +Y_{23} X_{34} X_{42}+X_{12} Z_{23} Y_{34} X_{41}-Z_{23} X_{34} X_{41} Y_{12}
\end{aligned}
$$

This is the superpotential for the gauge theory dual to the complex cone over the first del Pezzo surface $d P_{1}$. Note that we have relabelled some nodes for aesthetic reasons.

Now, let's see what happens when we set $\left\langle X_{45}\right\rangle \neq 0$. Since $X_{45}$ appears in a cubic term $X_{45} X_{53} X_{34}$ in the $d P_{2}$ superpotential, this vev has the effect of giving a mass to $X_{53}$ and $X_{34}$. These fields should then be integrated out of the IR theory. The classical equations of motion are

$$
\frac{\partial W}{\partial X_{53}}=X_{45}-Y_{31} X_{15}=0, \quad \frac{\partial W}{\partial X_{34}}=X_{53}-X_{42} Y_{23}=0 .
$$

The resulting superpotential is then purely quartic and is given by

$$
W_{\mathbf{F}_{0}}=X_{12} Y_{23} X_{34} Y_{41}-X_{12} X_{23} X_{34} X_{41}-Y_{12} Y_{23} Y_{34} Y_{41}+Y_{12} X_{23} Y_{34} X_{41},
$$

which is indeed the superpotential for $\mathbf{F}_{0}$. Notice that $d P_{2}$ has 11 fields, $d P_{1}$ has 10 , and $\mathbb{F}_{0}$ has 8 . The cubic term has had the effect of removing two additional fields from the spectrum, as it must.

The R-charges for this theory must be computed via $a$-maximization [12]. There are a priori 11 different R-charges, subject to 5 constraints from anomaly freedom and 6 constraints from the superpotential. One can easily check that there are, however, 4 undetermined R-charges; the maximization must then be done over the space of these 4 charges [13]. This will be a general feature of our new quivers.

Let's now recall the toric presentations of the complex cones over $\mathbb{F}_{0}, d P_{1}$, and $d P_{2}$. Since these cones are Calabi-Yau, we may represent the toric data with a two dimensional lattice. A perhaps familiar presentation of the toric data for these three surfaces is given in Figure 3. The toric diagram can be used to read off the number of 2- and 4-cycles in the corresponding Calabi-Yau cone: The number of internal points is the number of 4-cycles, and the number of 2-cycles can be derived from the requirement that $\#(0$-cycles $)+\#(2$-cycles $)+\#(4$-cycles $)=2$ (Area); this is the simply the Euler characteristic of the Calabi-Yau. This number is also the same as the number of gauge groups in the dual gauge theory. The Euler characteristic is also the number of gauge groups in the dual quiver theory, as one can check for these examples ${ }^{1}$. Since each space here is connected, the number of 0 -cycles is always 1 . It is then straightforward to figure out that the number of 2-cycles for $\mathbb{F}_{0}, d P_{1}$, and $d P_{2}$ is two, two, and three, respectively. This corresponds exactly with out geometric intuition: Since $\mathbf{F}_{0}=\mathbb{P}^{1} \times \mathbb{P}^{1}$, we expect two independent 2-cycles on $\mathbf{F}_{0}$, and since $d P_{n}$ is just $\mathbb{P}^{2}$ blown up at $n$ points, we expect $n+12$-cycles for $d P_{n}$.

\footnotetext{
${ }^{1}$ This rule was discovered empirically with B. Kol; the brane dimer picture 31, 32] provides a proof.
} 

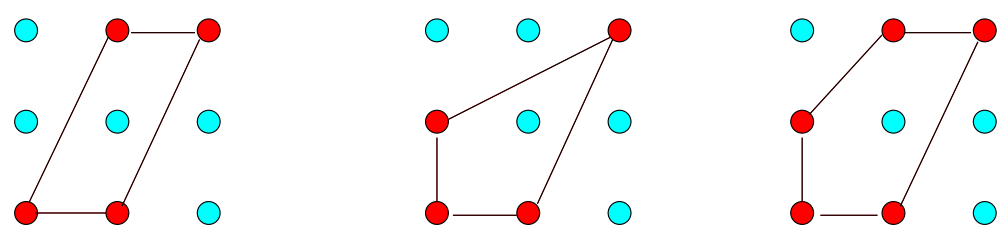

Figure 3: The toric data for $\mathbb{F}_{0}$ (left), $d P_{1}$ (center), and $d P_{2}$ (right).

Other (and for our purposes later on, more convenient) toric presentations of these spaces are also possible. For future reference, we include these alternate presentations in Figure 1 . One can easily check that these toric diagrams yield the same number of 2- and 4-cycles as the ones in Figure 3. The relation between the two toric presentations is simply that the points along the diagonal in Figure 3 have been brought to lie along a vertical line in Figure $4 ;$ the two toric diagrams correspond to different projections of the full three-dimensional toric diagram for the Calabi-Yau cones. The presentations of Figure 3 can be mapped to those of Figure 1 by an affine transformation, whose form we do not record here.
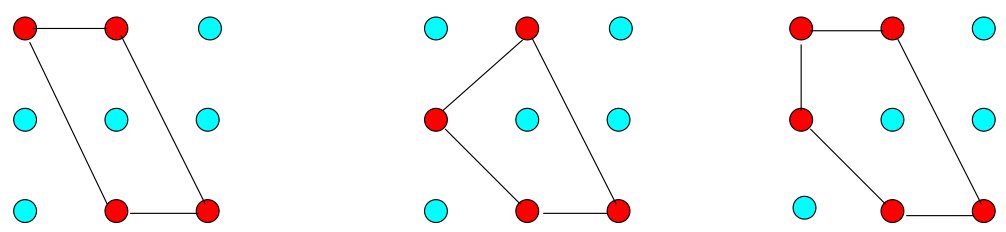

Figure 4: Alternate toric data for $\mathbb{F}_{0}$ (left) $d P_{1}$ (center), and $d P_{2}$ (right).

Note that it is possible to see Higgsing from the toric diagram. Since twice the area of the toric diagram (i.e. the number of triangles in a triangulation) is the number of gauge groups in the dual superconformal theory, Higgsing corresponds to removing an external point from the toric diagram. All the processes we consider here will never decrease the number of triangles by more than one, meaning that we only consider Higgsings that break $S U(N)^{m} \rightarrow S U(N)^{m-1}$ (cases for which this is not true are more mysterious and not well understood). In the example above, it's easy to see how the toric diagram for $d P_{2}$ can be altered to give the toric diagrams for $d P_{1}$ and $\mathbb{F}_{0}$ : To get $d P_{1}$, delete the top left point from the $d P_{2}$ diagram in Figure $4 ;$; to get $\mathbf{F}_{0}$, delete the other point on the left side of the toric diagram. Note that the external lines in the toric diagrams correspond to places where the $T^{3}$ fiber of the toric geometry has a degenerate cycle. Removing an external line is thus the same as blowing down a 2-cycle; this matches our intuition for how to get from $d P_{2}$ to $d P_{1}$ or $\mathbb{F}_{0}$. It is also possible to see this process from the corresponding (p,q)-brane web, which is straightforward to read off the toric diagram. We postpone the discussion of (p,q) webs until Section 4.

As a final warm-up example, we recall the theory that blows down to the gauge theories dual to the conifold and $S^{5} / \mathbb{Z}_{2}$. This theory is known as the Suspended Pinch Point (SPP), and was 
first described in [5]. This theory can be Higgsed to the simplest $Y^{p, q}$ spaces, $Y^{1,0}$ and $Y^{1,1}$. $Y^{1,0}$ is simply the conifold theory, and $Y^{1,1}$ is the $\mathcal{N}=2$ theory dual to $\mathbb{C}^{3} / \mathbb{Z}_{2}$. This Higgsing is illustrated in Figure 5 .

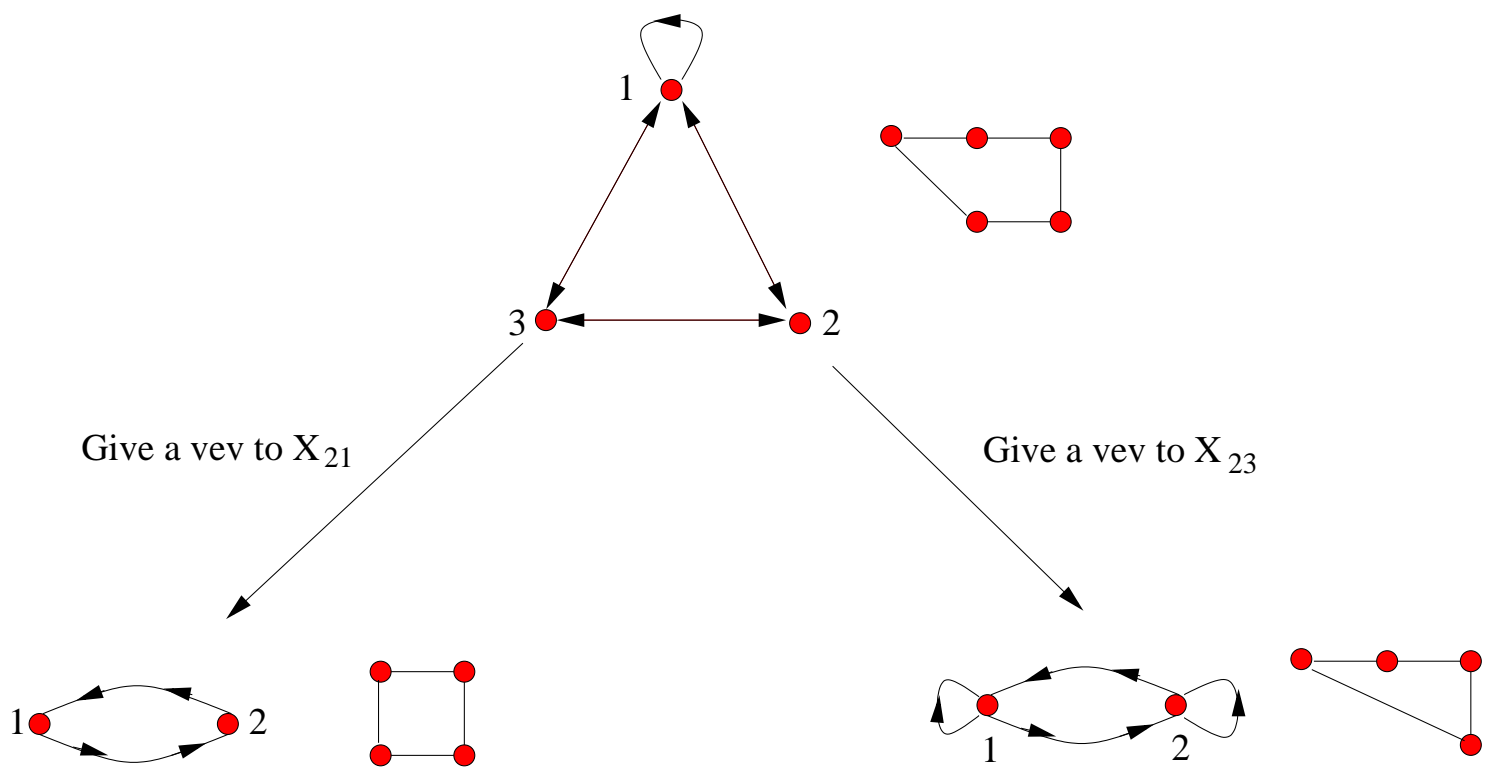

Figure 5: The SPP quiver (top) can be Higgsed to the conifold (bottom left) or the $\mathbb{C}^{3} / \mathbb{Z}_{2}$ theory (bottom right).

The superpotential for the SPP is given by

$$
W_{S P P}=X_{12} X_{23} X_{32} X_{21}-X_{23} X_{31} X_{13} X_{32}+X_{13} X_{31} X_{11}-X_{12} X_{21} X_{11} .
$$

It is easy to see that upon setting $\left\langle X_{23}\right\rangle \neq 0$, the superpotential becomes purely cubic. This is exactly as expected for the $\mathcal{N}=2$ theory, since it is an orbifold of $\mathbb{C}^{3}$. Giving $X_{21}$ a vev results in a mass term for the fields $X_{12}$ and $X_{11}$, which must then be integrated out. Doing so reproduces the superpotential for the conifold, given by two quartic terms.

\section{New quiver theories: $X^{p, q}$}

In this section we give a procedure for constructing quivers which blow down to $Y^{p, q}$ and $Y^{p, q-1}$. The general idea is to start with a known quiver gauge theory, say $Y^{p, q}$, blow up its corresponding toric diagram and then to find the effect on the quiver. This procedure was done for several examples in 33]. In many situations there is a unique way to perform such a blow up; this gives the resulting quiver gauge theory. It turns out that in the case we study here we have such a situation in which a blow up gives a unique answer and therefore allows the construction of a new infinite family of quiver gauge theories. We denote the quivers we construct in this paper by $X^{p, q}$; in this language, the $d P_{2}$ quiver would be called $X^{2,1}$, and the SPP would be $X^{1,1}$. We will see that 
the procedure for constructing $d P_{2}$ generalizes nicely to general $p$ and $q$. The blow-up we construct is the unique possiblity for blowing up the specified node, but of course one could always choose to blow up a different node. In a later section, we will show that blowing up a different node simply results in a theory which is Seiberg dual to the one presented here.

Before we continue on to the $X^{p, q}$ spaces, however, we briefly review the $Y^{p, q}$ quivers. This will be a quick discussion; for more details the reader is referred to [11].

\subsection{Review of the $Y^{p, q}$ quivers}

The $Y^{p, q}$ theories were constructed in [11], following the discovery of the dual geometries and their toric descriptions in [7, 8, 9]. It was shown there that they can be obtained as modifications of $Y^{p, p}$, which happens to be the theory living on a stack of D3 branes placed on the singular point of the orbifold $\mathbb{C}^{3} / \mathbb{Z}_{2} \times \mathbb{Z}_{p}$. The orbifold theory can be constructed by standard methods as a projection of $\mathcal{N}=4 \mathrm{SYM}$. The gauge group is $S U(N)^{2 p}$ where $N$ is the number of D3 branes in the stack. The $Y^{p, p}$ theory has $6 p$ bifundamental matter fields transforming in $2 p$ doublets $U^{\alpha}, \alpha=1,2$, and $2 p$ singlets $Y$ of the $S U(2)$ nonabelian part of the global symmetry group. The superpotential for

this theory descends from that of $\mathcal{N}=4$ and consists of $4 p$ cubic terms. The quiver for $Y^{3,3}$ can be seen in the top left corner of Figure 6. The superpotential for this theory is

$$
\begin{aligned}
W_{Y^{3,3}} & =\epsilon^{\alpha \beta} U_{12}^{\alpha} U_{23}^{\beta} Y_{31}+\epsilon^{\alpha \beta} U_{23}^{\alpha} U_{34}^{\beta} Y_{42}+\epsilon^{\alpha \beta} U_{34}^{\alpha} U_{45}^{\beta} Y_{53} \\
& +\epsilon^{\alpha \beta} U_{45}^{\alpha} U_{56}^{\beta} Y_{64}+\epsilon^{\alpha \beta} U_{56}^{\alpha} U_{61}^{\beta} Y_{15}+\epsilon^{\alpha \beta} U_{61}^{\alpha} U_{12}^{\beta} Y_{26} .
\end{aligned}
$$

The doublets are contracted in a way that respects the $S U(2)$ global symmetry.

To construct the $Y^{p, p-1}$ theory, one picks a doublet in $Y^{p, p}$, say the one between nodes $i$ and $i+1$, and removes one of the two bifundamentals. Then one removes the singlets $Y_{i+2, i}, Y_{i+1, i-1}$ and adds a new singlet $Y_{i+2, i-1}$. Four of the cubic terms in the superpotential are eliminated by these removals. Finally, one adds two quartic terms to the superpotential, involving the remaining of the two $U$ fields (now called $Z$ ), the new singlet and two $U$ doublets. As an example, the quiver for $Y^{3,2}$ is shown in the top right corner of Figure 6. The superpotential for this theory reads:

$$
\begin{aligned}
W_{Y^{3,2}} & =\epsilon^{\alpha \beta} U_{12}^{\alpha} U_{23}^{\beta} Y_{31}+\epsilon^{\alpha \beta} U_{23}^{\alpha} Z_{34} U_{45}^{\beta} Y_{52} \\
& +\epsilon^{\alpha \beta} U_{45}^{\alpha} U_{56}^{\beta} Y_{64}+\epsilon^{\alpha \beta} U_{56}^{\alpha} U_{61}^{\beta} Y_{15}+\epsilon^{\alpha \beta} U_{61}^{\alpha} U_{12}^{\beta} Y_{26} .
\end{aligned}
$$

This procedure repeated $p-q$ times for non-consecutive $U$ doublets yields $Y^{p, q}$. In the lower half of Figure 6 we show the quivers for $Y^{3,1}$ and $Y^{3,0}$. Each time $q$ descreases by one, four cubic terms are eliminated and two quartic terms appear in the superpotential. The superpotential of $Y^{p, q}$ therefore has $4 q$ cubics and $2(p-q)$ quartics. The modifications to the $Y^{p, p}$ quiver are called single impurities; we say that there is a single impurity between any two nodes where there is a 
bifundamental $Z$. The specific choice of sites on the quiver where single impurities are placed is not important, since the different theories obtained this way are related by Seiberg duality [18] and have the same IR dynamics. In fact, single impurities can be brought on top of each other and combine into double impurities, which contribute cubic terms to the superpotential. We shall say more about these in a later section.
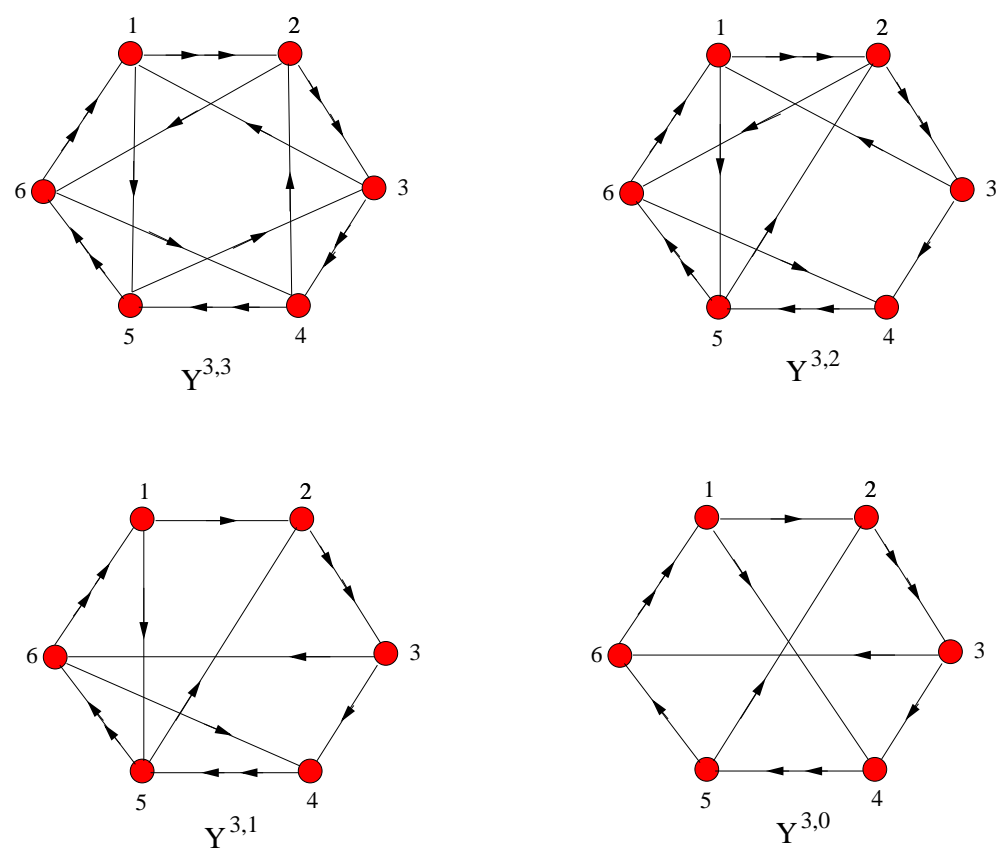

Figure 6: Quivers for the $Y^{3, q}$ theories.

\section{$3.2 X^{p, q}$ for $1 \leq q \leq p-1$}

Now that we have reviewed the $Y^{p, q}$ theories, constructing the $X^{p, q}$ quivers is straightfoward. We first consider the case $0 \leq q \leq p-1$, since the procedure we give here must be altered slightly when $q=p$; this latter case will be described subsequently. Consider the quiver for $Y^{p, q}$. One toric phase of this theory will have $p-q$ single impurities, where double arrows on the outside of the $Y^{p, p}$ quiver have been replaced with single arrows. Since we only consider cases where $q \leq p-1$, there will always be at least one single arrow on the outside of the $Y^{p, q}$ quiver. Without loss of generality, we can choose this arrow to be as close as possible to the leg which is impurified when constructing the $Y^{p, q-1}$ quiver; if the single arrow is farther away, it is always possible to perform a sequence of Seiberg dualities to bring it to the desired position. See Figure 7 .

Let's call the node we blow up node $B$, which we blow up into the two nodes $B_{1}$ and $B_{2}$. Denote the node before $B$ by $A$, and the node after $B$ by $C$. The new $X^{p, q}$ quiver is constructed as follows:

- Draw bifundamentals $X_{A B_{1}}, X_{B_{1} B_{2}}, X_{B_{2} C}, X_{A B_{2}}, X_{B_{1} C}$. 

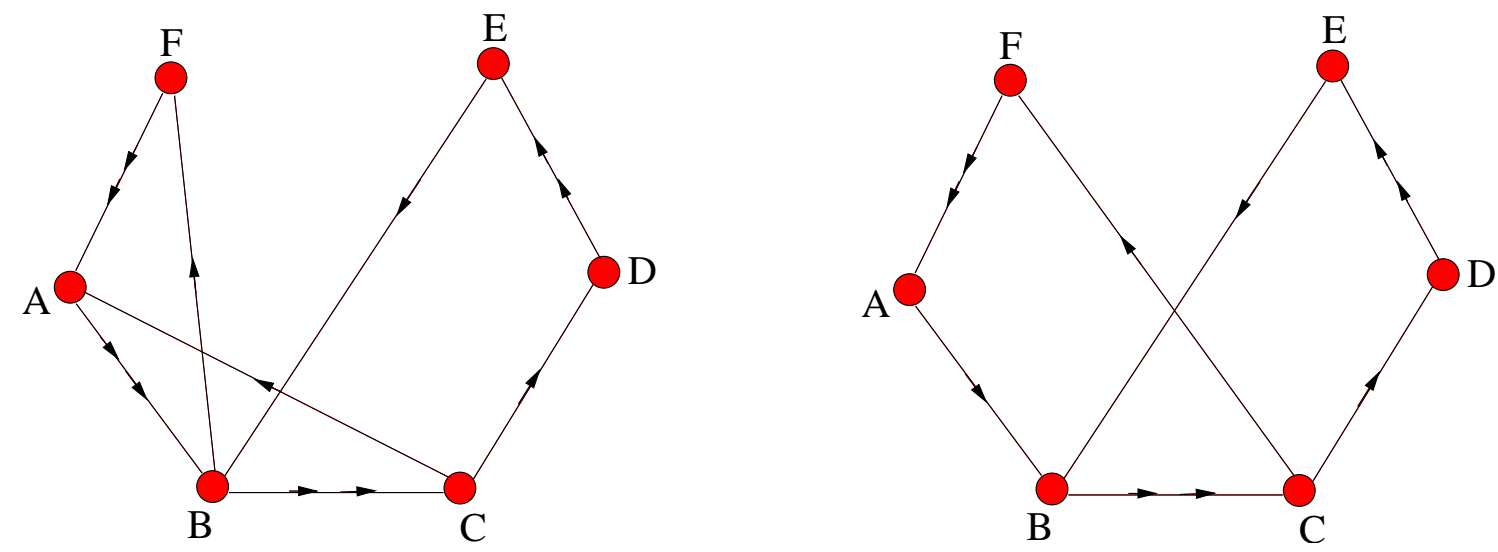

Figure 7: The relevant portions of the quivers for $Y^{p, q}$ (left) and $Y^{p, q-1}$ (right). The rest of the quiver is assumed to be in between nodes $\mathrm{E}$ and $\mathrm{F}$ and is not drawn.

- For all single bifundamentals in the $Y^{p, q}$ quiver of the form $X_{n B}$ (i.e. entering $B$ ), draw a bifundamental $X_{n B_{1}}$.

- For all single bifundamentals in the $Y^{p, q}$ quiver of the form $X_{B n}$ (i.e. exiting $B$ ), draw a bifundamental $X_{B_{2} n}$.

- All other bifundamentals should be left as they are in the $Y^{p, q}$ quiver.

For a graphical depiction of this process, see Figure 8 .

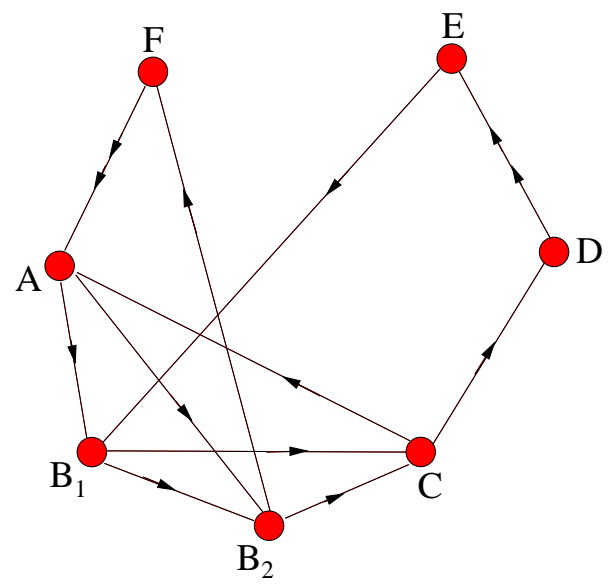

Figure 8: The relevant portions of the quivers for $Y^{p, q}$ (left) and $Y^{p, q-1}$ (right).

Notice that there are $4 p+2 q$ bifundamentals in the $Y^{p, q}$ quiver. Four of them are the double arrows entering and exiting node $B$, which get replaced with five single arrows in the $X^{p, q}$ quiver. Thus there is a net increase of one in the number of arrows, meaning that this phase of our $X^{p, q}$ theories will always have $4 p+2 q+1$ bifundamentals. As we saw in the previous section, this is 
exactly the case with $d P_{2}$, which has 11 fields, compared to the 10 fields of $d P_{1}$ and the 8 fields of $\mathbb{F}_{0}$.

Obtaining the superpotential is straightforward. We know that in the superpotential for a $Y^{p, q}$ quiver theory, there are $4 q$ cubic terms and $2(p-q)$ quartic terms. To reproduce this upon Higgsing, we must have the superpotential

$$
\begin{aligned}
W_{X^{p, q}} & =U_{D E}^{(2)} Y_{E B_{1}} Z_{B_{1} B_{2}} Z_{B_{2} C} Z_{C D}+Z_{A B_{1}} Y_{B_{1} C} Y_{C A}+U_{F A}^{(2)} Y_{A B_{2}} Y_{B_{2} F} \\
& -Y_{E B_{1}} Y_{B_{1} C} Z_{C D} U_{D E}^{(1)}-Y_{A B_{2}} Z_{B_{2} C} Y_{C A}-U_{F A}^{(1)} Z_{A B_{1}} Z_{B_{1} B_{2}} Y_{B_{2} F} \\
& + \text { unchanged, }
\end{aligned}
$$

where "unchanged" simply denotes all the other terms in the original $Y^{p, q}$ superpotential, which are unaffected by the blowup. In this sense, blowing up a node is a "local" procedure - it only affects the fields within 3 nodes of the blown up node.

That the $X^{p, q}$ quivers Higgs to $Y^{p, q}$ and $Y^{p, q-1}$ is now easy to see. Setting $\left\langle Z_{B_{1} B_{2}}\right\rangle \neq 0$ collapses the nodes $B_{1}$ and $B_{2}$ back into node $B$. We lose the field $Z_{B_{1} B_{2}}$ and the other fields remain, although we should rewrite any $B_{1}$ and $B_{2}$ indices as $B$. This gives (as it should, by our construction), the $Y^{p, q}$ quiver. The superpotential (3.5) also does exactly what it must. Setting $\left\langle Z_{B_{1} B_{2}}\right\rangle \neq 0$ changes the quintic term into a quartic, and one of the quartics into a cubic. This gives the superpotential for $Y^{p, q}$, where it is obvious that the global $S U(2)$ symmetry has been restored: The doublets are $\left(Z_{A B_{1}}, Y_{A B_{2}}\right),\left(Y_{B_{1} C}, Z_{B_{2} C}\right),\left(U_{F A}^{(1)}, U_{F A}^{(2)}\right)$, and $\left(U_{D E}^{(1)}, U_{D E}^{(2)}\right)$.

Giving a vev to $Z_{B_{2} C}$ yields the quiver for $Y^{p, q-1}$. This affects the superpotential in a mildly more nontrivial way than the previous case, since now the fields $Y_{A B_{2}}$ and $Y_{C A}$ get a mass and should be integrated out. Since these two fields appear in only cubic terms, the net effect of integrating them out is to replace the three cubic terms with one quartic. This quartic is exactly what we'd expect; it is paired with $U_{F A}^{(1)} Z_{A B_{1}} Z_{B_{1} B_{2}} Y_{B_{2} F}$ under the newly restored $S U(2)$ symmetry. For completeness, we note that the new $S U(2)$ doublets are given by $\left(U_{F A}^{(1)}, U_{F A}^{(2)}\right),\left(U_{D E}^{(1)}, U_{D E}^{(2)}\right)$, and $\left(Z_{B_{1} B_{2}}, Y_{B_{1} C}\right)$.

We note here that the total number of terms of a given degree in the superpotential for $X^{p, q}$ is easy to figure out. There is always one quintic, $2(p-q)$ quartics, and $4 q-1$ cubic terms. The reason is clear: In the $Y^{p, q}$ quiver, there are $2(p-q)$ quartics and $4 q$ cubics. Since blowing down the $X^{p, q}$ quiver to $Y^{p, q}$ involves shifting a quintic to a quartic and a quartic to a cubic, we see that the number of quintic terms in the $X^{p, q}$ superpotential is one, whereas the net number of cubic terms decreases by one and the net number of quartics remains the same.

\section{$3.3 X^{p, q}$ for $q=p$}

Now, let's consider the case $q=p$. The above procedure clearly must be modified, since a quintic term in the superpotential may no longer exist since there is nothing for it to descend to in the $Y^{p, p}$ theory. Nevertheless, the procedure is more or less the same as above, the only difference being 
that instead of drawing a bifundamental between node $E$ and node $B_{1}$, we draw one between $D$ and $B_{1}$. This is shown in Figure 9; as before, the parts of the quiver that do not change are not shown.

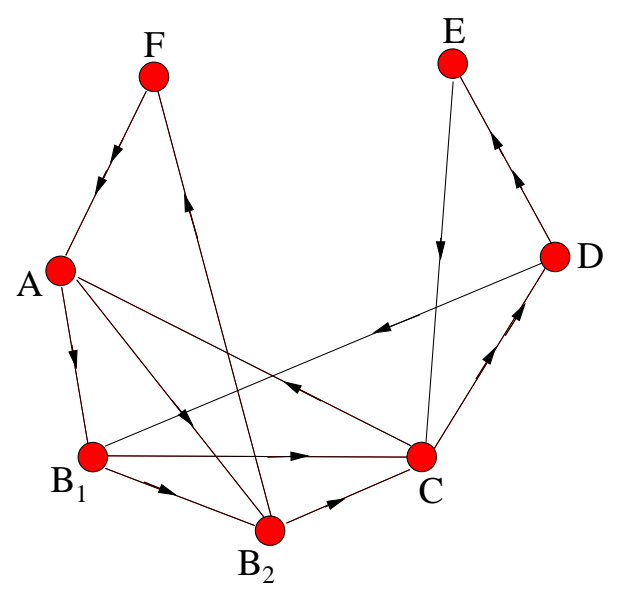

Figure 9: The relevant portions of the quivers for $Y^{p, q}$ (left) and $Y^{p, q-1}$ (right).

The superpotential is now given by

$$
\begin{aligned}
W_{X} X^{p, q} & =Z_{B_{2} C} Y_{C A} Y_{A B_{2}}+U_{C D}^{(2)} Y_{D B_{1}} Y_{B_{1} C}-Y_{B_{1} C} Y_{C A} Z_{A B_{1}}-Y_{A B_{2}} Y_{B_{2} F} U_{F A}^{(2)} \\
& -Z_{B_{2} C} U_{C D}^{(1)} Y_{D B_{1}} Z_{B_{1} B_{2}}+Z_{B_{1} B_{2}} Y_{B_{2} F} U_{F A}^{(1)} Z_{A B_{1}} \\
& + \text { unchanged, }
\end{aligned}
$$

Notice that, as before, when we set $\left\langle Z_{B_{1} B_{2}}\right\rangle \neq 0$, the theory flows to $Y^{p, p}$, and when we set $\left\langle Z_{B_{1} C}\right\rangle \neq 0$, the theory flows to $Y^{p, p-1}$. In the latter case, the fields $Y_{A B_{2}}$ and $Y_{A C}$ acquire a mass and should be integrated out; this yields the correct superpotential for $Y^{p, p-1}$.

For general $p$, then, we see that this $X^{p, p}$ theory has $6 p+1$ fields. The superpotential has 2 quartic terms and $4 p-2$ cubics. Going to the $Y^{p, p}$ theory simply changes both quartics into cubics, which recovers the $4 p$ cubic terms required for this theory. Flowing to the $Y^{p, p-1}$ theory involves shifting one quartic to a cubic, and taking three cubics into one quartic. Thus, the resulting theory has two quartic terms and $4(p-1)$ cubics, which is correct for $Y^{p, p-1}$.

It is worth pointing out again that the above prescription gives merely one way of constructing the $X^{p, q}$ theories, and there are many different possible toric phases of these theories. We will explore these dualities in a later section.

3.4 An example: $X^{3,1}, X^{3,2}$, and $X^{3,3}$

As an illustrative example, we now present quivers for $X^{3,1}, X^{3,2}$, and $X^{3,3}$. These theories will Higgs to the quivers for $Y^{3,0}, Y^{3,1}, Y^{3,2}$, and $Y^{3,3}$ in Figure 6. Apply the procedure outlined in the 
previous sections is straightforward, and yields the quivers in Figure 10.
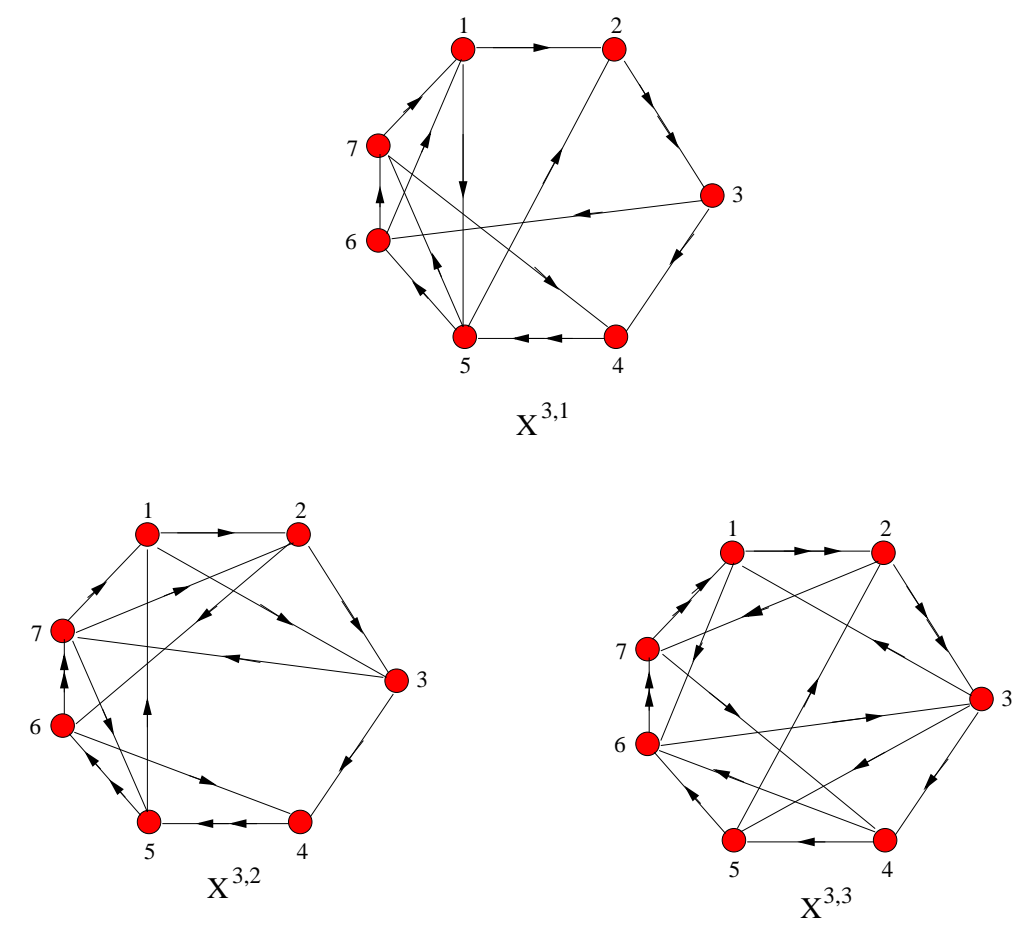

Figure 10: Quivers for a particular phase of $X^{3,1}, X^{3,2}$, and $X^{3,3}$.

We can easily write down the superpotentials for these theories. They are

$$
\begin{aligned}
W_{X^{3,1}} & =U_{23}^{(2)} Y_{36} Z_{67} Z_{71} Z_{12}+Z_{56} Y_{61} Y_{15}+U_{45}^{(1)} Y_{57} Y_{74} \\
& -Y_{36} Y_{61} Z_{12} U_{23}^{(1)}-Y_{57} Z_{71} Y_{15}-U_{45}^{(2)} Z_{56} Z_{67} Y_{74} \\
& +\epsilon^{\alpha \beta} U_{23}^{\alpha} Z_{34} U_{45}^{\beta} Y_{52}, \\
W_{X^{3,2}} & =U_{45}^{(2)} Y_{51} Z_{12} Z_{23} Z_{34}+Z_{71} Y_{13} Y_{37}+U_{67}^{(1)} Y_{72} Y_{26} \\
& -Y_{51} Y_{13} Z_{34} U_{45}^{(1)}-Y_{72} Z_{23} Y_{37}-U_{67}^{(2)} Z_{71} Z_{12} Y_{27} \\
& +\epsilon^{\alpha \beta} U_{45}^{\alpha} U_{56}^{\beta} Y_{64}+\epsilon^{\alpha \beta} U_{56}^{\alpha} U_{67}^{\beta} Y_{71},
\end{aligned}
$$

and

$$
\begin{aligned}
W_{X^{3,3}} & =Z_{56} Y_{63} Y_{35}+U_{67}^{(2)} Y_{74} Y_{46}-Y_{46} Y_{63} Z_{34}-Y_{35} Y_{52} U_{23}^{(2)} \\
& -Z_{56} U_{67}^{(1)} Y_{74} Z_{45}+Z_{45} Y_{52} U_{23}^{(1)} Z_{34} \\
& +\epsilon^{\alpha \beta} U_{67}^{\alpha} U_{71}^{\beta} Y_{16}+\epsilon^{\alpha \beta} U_{71}^{\alpha} U_{12}^{\beta} Y_{27}+\epsilon^{\alpha \beta} U_{12}^{\alpha} U_{23}^{\beta} Y_{31} .
\end{aligned}
$$


As they must, these become the $Y^{3, q}$ superpotentials upon giving vevs to the appropriate fields and integrating out where necessary.

\subsection{Toric Diagrams for $X^{p, q}$}

We now describe the toric presentation of the $X^{p, q}$ spaces. As discussed in the introduction, it is a rare occurrence to know the toric diagram corresponding to a given quiver. The Forward Algorithm [10] can be used to extract the toric data for simple quivers, but it is computationally prohibitive for quivers with many nodes. So knowing the toric data dual to an infinite number of quivers is highly nontrivial. Finding the toric data for the $X^{p, q}$ theories is straightforward, since we can use our intuition from $d P_{2}$ to simply write down the answer and then check that it is correct. First, we note two different toric presentations of the space $Y^{p, q}$; these are given in Figure 11. The toric data on the left is the presentation used in [9, 11]; the toric data on the right is simply an alternate projection which is particularly useful for our purposes ${ }^{2}$. Notice that for a given $p$, the only point that moves is the point along the left edge of the lattice. As $q$ decreases, this point moves up; at $q=0$ we recover the expected parallelogram for $Y^{p, 0}$.
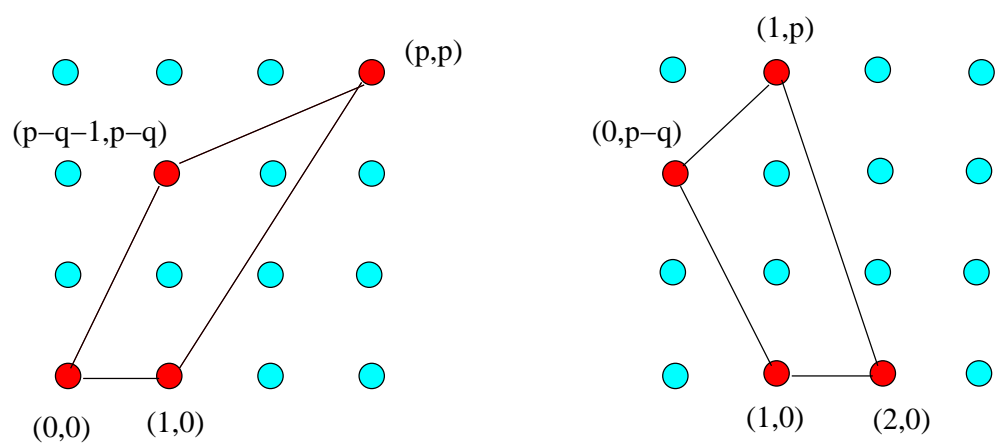

Figure 11: Two different presentations of the toric data for $Y^{p, q}$. The figures here are $Y^{3,1}$.

The toric data for $X^{p, q}$ is now easy to intuit. Since we need a space which blows down to both $Y^{p, q}$ and $Y^{p, q+1}$, we simply include both points on the left edge of the lattice, as in Figure 12. Removing the top left point leaves the toric diagram for $Y^{p, q}$, and removing the one below it yields the toric diagram for $Y^{p, q-1}$. Note that, as for $Y^{p, q}$, these two points move up as $q$ decreases.

There are many checks that these are the correct toric diagrams dual to the $X^{p, q}$ quivers. First, one may use the Forward Algorithm of [10] to extract the toric data. These have been checked for small $p$, and yield the expected results ${ }^{3}$. We may also check the number of gauge groups, which is equal to the number of triangles in a triangluation of the toric diagram. This should be equal to $2 p+1$ in general, and it is straightforward to see that this is correct: The $Y^{p, q}$ theories have $2 p$ triangles, and the effect of adding the extra node is to add one more. Additionally, we can read off

\footnotetext{
${ }^{2}$ This representation was also used in [14].

${ }^{3}$ We thank Alan Dunn for this calculation.
} 


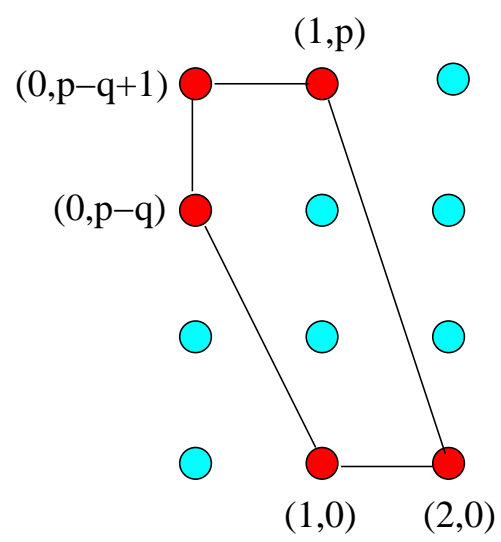

Figure 12: The toric data for $X^{p, q}$. This diagram is for $X^{3,1}$, which blows down to $Y^{3,0}$ and $Y^{3,1}$.

the even Betti numbers of the Calabi-Yau cone. There are $p-1$ internal points, corresponding to $p-14$-cycles, and since the space is connected there is only one 0-cycle. Therefore, there must be $p+12$-cycles. Thus, the number of 2-cycles for the $X^{p, q}$ theories is one greater than it is in the lower $Y^{p, q}$ theories, as we expect by analogy to $d P_{2}$.

Notice that we can rephrase the above as follows: If $I$ is the number of internal points in the toric diagram and $E$ is the number of external points, then the number of 4-cycles is $I$, and the number of 2-cycles is $I+E-3$. This has an interpretation in the related five dimensional gauge theory, as we will discuss in the next section.

We also can find some properties of the corresponding Sasaki-Einstein manifold at the base of the Calabi-Yau cone ${ }^{4}$. For a Sasaki-Einstein space whose toric diagram has $d$ external lines, $H_{3}=\mathbb{Z}^{d-3}$, so here we find $H_{3}\left(X^{p, q}\right)=\mathbb{Z}^{2}$. Thus there are always two 3 -cycles available for a D3-brane to wrap; this will be discussed further in Section 6. The topological possibilities for fivedimensional spaces are known, thanks to Smale's Theorem. Here, we can say that the $X^{p, q}$ SasakiEinstein manifolds for $p \neq q$ (the $p=q$ case is singular) are a connected sum $\left(S^{2} \times S^{3}\right) \#\left(S^{2} \times S^{3}\right)$.

Knowing that the toric diagram in Figure 12 gives a Calabi-Yau dual to the $X^{p, q}$ theories is highly nontrivial. Although our construction of the gauge theories is done without ever considering the dual geometry, it is important to point out that we know information about both sides of the AdS/CFT duality. The metrics on the $X^{p, q}$ geometries are not known, and appear to be quite difficult to calculate. The $Y^{p, q}$ theories had a global $S U(2)$ symmetry; the existence of this nonAbelian symmetry was crucial to figuring out the metrics on the Sasaki-Einstein manifolds [7]. The $X^{p, q}$ gauge theories have only $U(1)$ global symmetries, so we lose the power of the non-Abelian isometry when trying to find the dual metrics. Thus, the Sasaki-Einstein metrics on these spaces are unknown, and probably rather difficult to derive.

\footnotetext{
${ }^{4}$ We thank James Sparks for discussions on this.
} 


\section{4. $(\mathrm{p}, \mathrm{q})$-brane webs and $5 \mathrm{~d}$ gauge theories}

It is known that one can get a five-dimensional theory associated to the theory living on the D3-branes at the tip of the Calabi-Yau cone by writing down a web of $(p, q)^{5} 5$-branes [26]; the procedure for deriving the brane web from the corresponding toric diagram is well-known [23, 25]. More mysterious, however, is what one can say about the resulting 5-dimensional gauge theory living on the $4+1$ dimensions common to all the 5-branes. Some things are known about the correspondence between the five dimensional theory and the related four dimensional quiver [34], but much still remains unkown. Let us now briefly review what is known about (p,q)-webs and the associated 5 dimensional gauge theories.

Type IIB string theory has a vast armada of $5+1$ dimensional branes, the (p,q) 5-branes. (p,q) 5-branes are bound states of different numbers of D5-branes and NS5-branes; we take the convention that a $(1,0)$ brane is a D5-brane, and a $(0,1)$ brane is an NS5-brane. A (p,q) 5-brane is simply the magnetic dual of a $(p, q)$ string, and may be thought of as coming from an M5-brane wrapped on a (p,q)-cycle of a $T^{2}$. The tension of an arbitrary (p,q) brane is then given by $T_{(p, q)}=|p+\tau q| T_{D 5}$, where $\tau$ is the Type IIB axion-dilaton. These (p,q) 5 -branes are useful tools for studying 5 d gauge theories, via arranging the branes in a network such that they share $4+1$ dimensions. The remaining dimension can be taken to lie in a plane, and the branes can be arranged in a network called a (p,q) web. Of course, placing branes at generic angles will break all supersymmetry. The requirement that a web be stable and preserve supersymmetry can be summed up in the conditions

$$
\frac{\Delta x}{\Delta y}=\frac{p}{q} \quad \text { and } \quad \sum_{i} p_{i}=\sum_{i} q_{i}=0 .
$$

In (4.1), the first condition states that the slope of a brane in the $(x, y)$ plane is equal to the ratio of its $(\mathrm{p}, \mathrm{q})$ charges, and the second condition is simply conservation of $\mathrm{p}$ and $\mathrm{q}$ charge at each vertex (the sum is over all branes ending at a given vertex). The slope condition ensures that one quarter of the supersymmetry is preserved, giving the 8 supercharges for a five-dimensional $\mathcal{N}=1$ theory.

It is now well-known that one can associate a toric diagram to a $(\mathrm{p}, \mathrm{q})$ web via a straightforward procedure, which we now review. First, one needs to pick a triangulation of the toric diagram; we have done this for $d P_{1}$ in Figure 13. The brane web is now essentially just the dual of this diagram: To construct it, just draw the lines orthogonal to the lines in the triangulated toric diagram. External lines in the toric diagram correspond to semi-infinite branes, and internal lines correspond to branes with finite extent in the $(x, y)$-plane. Notice that a consequence of this is that the number of internal points in the toric diagram corresponds to the number of closed polygons in the brane web.

As a warm-up for finding the $X^{p, q}$ brane webs, let's see how one can use the web for $d P_{2}$ to get the associated webs for $d P_{1}$ and $\mathbb{F}_{0}$. Related discussion can be found near Figure 12 of [26]. The

\footnotetext{
${ }^{5}$ Here we run into the problem of using the grouping $(\mathrm{p}, \mathrm{q})$ in two different contexts. (p,q) for 5 -branes will always be in Roman, and $(p, q)$ for $Y^{p, q}$ will be in math (italic).
} 

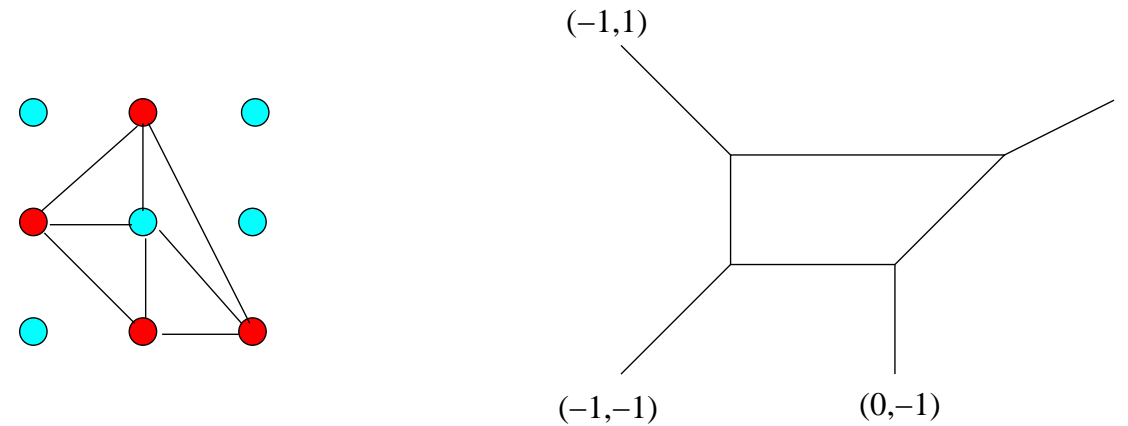

Figure 13: A triangulation of the toric data for $d P_{1}$ and the corresponding brane web.

brane webs for $d P_{2}, d P_{1}$, and $\mathbf{F}_{0}$ are shown in Figure 14; we have used the toric data of Figure 1 to construct them. Notice that the $d P_{2}$ web has a semi-infinite horizontal brane, which we have drawn in red. This is a D5-brane, and shows up in the $5 \mathrm{~d}$ theory as a massive flavor. As we move this brane up or down, the mass of the flavor changes and it may then be integrated out of the theory. Interestingly, the resulting theory is different depending how one increases the mass: By moving the $(-1,0)$ D5-brane up, it hits the $(0,1)$ brane and results in a $(-1,1)$ brane, giving the web for $d P_{1}$. By moving the D5-brane down, it hits the $(-1,-1)$ brane at the bottom and results in a $(-2,-1)$ brane, giving the $\mathbf{F}_{0}$ brane web. We will see analogous behavior in the $X^{p, q}$ brane webs when we generate the two possible descendant $Y^{p, q}$ webs.

We now see that knowing the toric diagram is tantamount to knowing the brane web. One can easily see in Figure 12 that we'll always get a flavor D5-brane which can be moved up or down; this is just the external line dual to the one external vertical line in the toric diagram. We do note that the situation is mildly more complicated for many internal points, since moving the flavor D5 past any brane junction means that one must change the $(\mathrm{p}, \mathrm{q})$ charges of the branes at the junction. One example of this procedure is done, for $X^{3,1}$, in Figure 15.

We also note here that integrating out the massive flavor is equivalent, geometrically, to blowing down a 2-cycle. This is especially simple to see in the $d P_{2}$ example, since we know that $d P_{2}$ blows down to either $d P_{1}$ or $\mathbb{F}_{0}$. This is also easy to see from the toric diagram, since external lines correspond to 2-cycles. For the general $X^{p, q}$ theoriesthis interpretation of blowing down a 2-cycle remains true. For an interesting discussion of the transitions between some of the $X^{p, q}$ and $Y^{p, q}$ theories, see Figure 25 of [25] and the surrounding discussion.

The $d P_{2}$ theory, or $X^{2,1}$ as we call it in this paper, corresponds to a five dimensional $S U(2)$ gauge theory with one flavor. Taking the mass of this flavor to positive infinity yields one of two different theories: If we define the sign of the mass to correspond to the positive $y$ direction in Figure 14, this theory is $\mathbb{F}_{0}$. Taking the mass to negative infinity gives the other theory, $d P_{1}$. The distinction between these two theories is related to the value of the discrete $\theta$ angle, which follows from the fact that the 4 th homotopy group of the gauge group is $\pi_{4}(S U(2))=\mathbb{Z}_{2}$. This distinction 


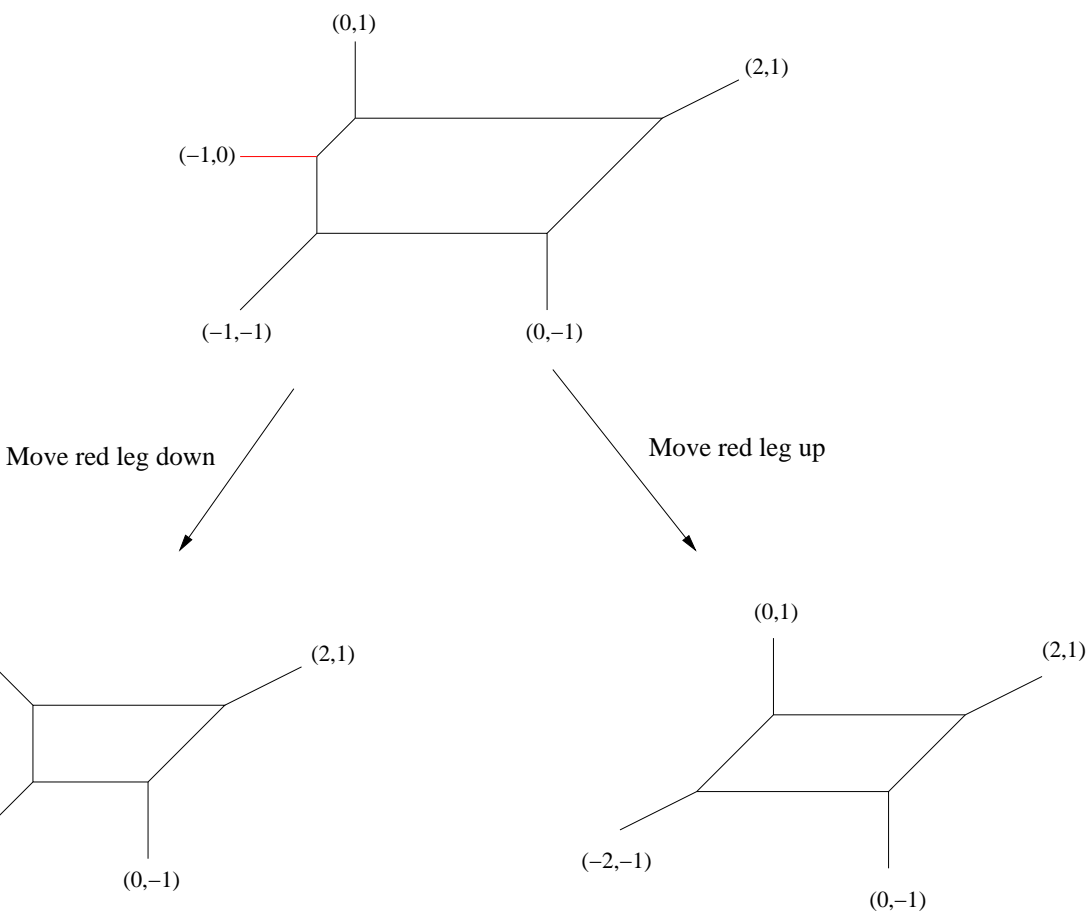

Figure 14: By moving the horizontal (red) brane in the above $d P_{2}$ brane web up or down, one can get a brane web for $d P_{1}$ (left) or $\mathbb{F}_{0}$ (right).

is special to the case of $S U(2)$ as this is the only unitary group with a non-trivial 4th homotopy group. For higher values of the rank of the unitary group the five dimensional theory can admit a Chern-Simons term. This term is absent for the $S U(2)$ case since the completely symmetric rank 3 invariant for $S U(2)$ vanishes, while for the other $S U(p)$ theories it is non-vanishing. As a result, for the $5 \mathrm{~d}$ theories living on the brane webs dual to the $Y^{p, q}$ toric diagrams with $p>2$ we can introduce a Chern-Simons term which turns out to have a coefficent equal to $q$. As discussed in detail in [35] a massive flavor contributes at the one loop level to the effective Chern-Simons coupling a value of $1 / 2$ and the sign of this contribution is proportional to the sign of the mass of this flavor. As a result when going from a large positive mass to a large negative mass and vice versa, the value of the Chern-Simons coefficient changes by 1. As we identify the Chern-Simons coupling with $q$, taking the mass from one sign to another precisely maps to the process of changing $q$ by 1 . This is the process we have discussed above in which we start with $X^{p, q}$, corresponding to a small mass in the 5d theory, and Higgs to either $Y^{p, q}$ or $Y^{p, q-1}$ by giving a large positive or negative mass, respectively, to the $5 \mathrm{~d}$ flavor.

One more interesting point to note about the $Y^{p, q}$ theories is that from a five dimensional point of view, the allowed values for $p$ and $q$ which give interacting UV fixed points in five dimensions are $p>q \geq 0$. The case with $q=0$ is the simplest five dimensional SYM $S U(p)$ gauge theory where there is no Chern-Simons term. The case with $q=p$ does not lead to an interacting UV 

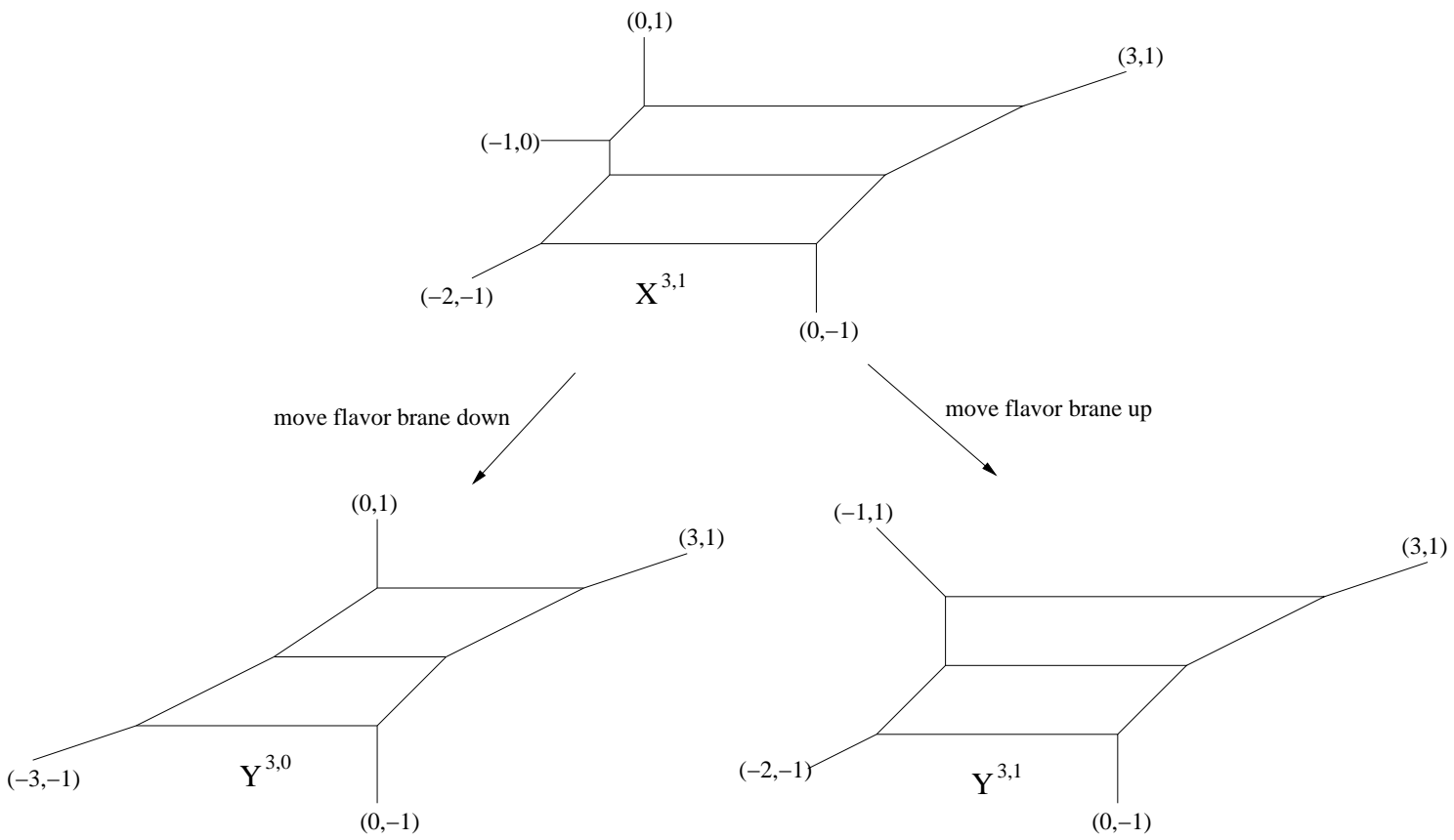

Figure 15: The process of going from the $X^{3,1}$ web to that of $Y^{3,0}$ or $Y^{3,1}$.

fixed point since the brane web involves parallel legs; see a discussion in [25]. The conditions which were written in [35] for an interacting fixed point coincide nicely with the allowed range of the $Y^{p, q}$ theories as required by the geometry side to have smooth metrics. These conditions can be extended to the case of more flavors. If we denote the number of flavors of the five dimensional gauge theory by $N_{f}$ then this number is related to the number of external nodes $E$ in the corresponding toric diagram by $E=N_{f}+4$. The condition for an interacting UV fixed point is then $N_{f}+2 q<2 p$. For $N_{f}=0$ this recovers the known limits on $Y^{p, q}$ discussed above while for $N_{f}=1$ we get a new bound which is consistent with the limits that we have found in this paper. We further get a prediction for the allowed ranges of theories for higher values of $N_{f}$.

As discussed in [25], for a given five dimensional gauge theory with $S U(p)$ gauge group and for any Chern-Simons coefficient $q$ the number of parameters in the Lagrangian is the number of external legs $E$ in the (p,q) web minus 3. As an example, for $N_{f}=0$ the number of parameters is 1 ; this parameter is simply the gauge coupling of the five dimensional gauge theory. For $N_{f}=1$ there is an additional parameter given by the mass of the flavor, etc. This number $E-3$ actually counts the number of baryonic $U(1)$ global symmetries in the corresponding quiver gauge theory. Thus for the $Y^{p, q}$ theories we have one $U(1)_{B}$ and for $X^{p, q}$ we expect two baryonic $U(1)$ global symmetries.

There are additional matchings we can make between the five dimensional theory and the quiver gauge theory. The number of moduli for the five dimensional theory is equal to the rank of the gauge group, $p-1$. This number gives the number of distinct monopole solutions in the five 
dimensional gauge theory as well as the various vacuum expectation values which can spontaneously break gauge invariance. For the geometry this number is the number of internal points in the toric diagram and is therefore the number of vanishing 4-cycles for the singular geometry. The number of 2-cycles in the singular geometry has yet another simple expression as $p-1+E-3$. This number also counts the number of different BPS charges $B$ that particles can carry in the five dimensional theory. As is well known the number of gauge groups for the quiver gauge theory is given by the total number of all even (0-,2-,and 4-) dimensional cycles. Therefore we get a relation which states that the number of different monopole solutions, denoted $M$, is given in terms of $p$ and the number of external lines as $M+B+1=2 M+N_{f}+2=2 M+E-2=2 p+N_{f}$. For the cases $N_{f}=0$ and $N_{f}=1$ we recover the known cases of $Y^{p, q}$ and $X^{p, q}$, respectively. See related discussions in [25]. We summarize the above discussion in Table 11.

\begin{tabular}{|c|c|}
\hline $5 \mathrm{~d} \mathrm{SU}(\mathrm{p})$ theory & $Y^{p, q}$ Toric Geometry \\
\hline \hline Number of Monopoles M & $p-1$ \\
\hline Number of BPS States B & Number of $2-$ cycles, $p-1+E-3$ \\
\hline $5 \mathrm{~d}$ Moduli & Number of $4-$ cycles, $p-1$ \\
\hline Chern - Simons Coefficient & $q$ \\
\hline Number of Flavors $N_{f}$ & $E-4$ \\
\hline
\end{tabular}

Table 1: The relationship between different parameters in the $5 \mathrm{~d}$ theories and $Y^{p, q}$ toric geometries.

\section{Toric Phases of the $X^{p, q}$ theories}

The $X^{p, q}$ quiver gauge theories we present in this paper each flow to a superconformal fixed point at the infrared. At that point one can apply Seiberg duality [36 to any of the gauge groups and get a new theory which has a different matter content and superpotential but flows to the same IR fixed point as the original theory. By repeating this process it is possible to obtain an infinite number of UV inequivalent theories that fall into the same universality class. In this section we will discuss a particularly simple finite subclass of these theories, the connected toric phases. We define the term toric phase to mean that all the gauge groups have the same rank. The term "connected" refers to the theories generated by starting from a toric phase, like the ones discussed above, and only dualizing nodes with number of flavors $\left(N_{f}\right)$ equal to twice the number of colors $\left(N_{c}\right)$. These are sometimes called self-dual nodes, because the gauge group is unchanged by the dualization. The resulting gauge theory is again in a toric phase, with gauge group $S U(N)^{2 p+1}$ and every field appearing in the superpotential exactly twice. For the sake of brevity we will omit 
the tedious details of the dualizations. Instead we shall outline the general structure of these toric phases, using a few of the results of [18] for the corresponding $Y^{p, q}$ quivers.

The toric phases of the $Y^{p, q}$ theories can be described as modifications of the quiver for $Y^{p, p}$. These can be seen very clearly in the "ladder" depiction of the quiver, in which the nodes are placed in two parallel rows and numbered in a crenellating ${ }^{6}$ fashion. The quiver is then made up of "blocks," i.e. square sections between rungs of the ladder. All the blocks are identical in $Y^{p, p}$ and each one can be replaced by a single or double impurity. The numbers of single impurities $\left(n_{1}\right)$ and double impurities $\left(n_{2}\right)$ are restricted by the relation $n_{1}+2 n_{2}=p-q$. An example of this construction is shown in Figure 16.

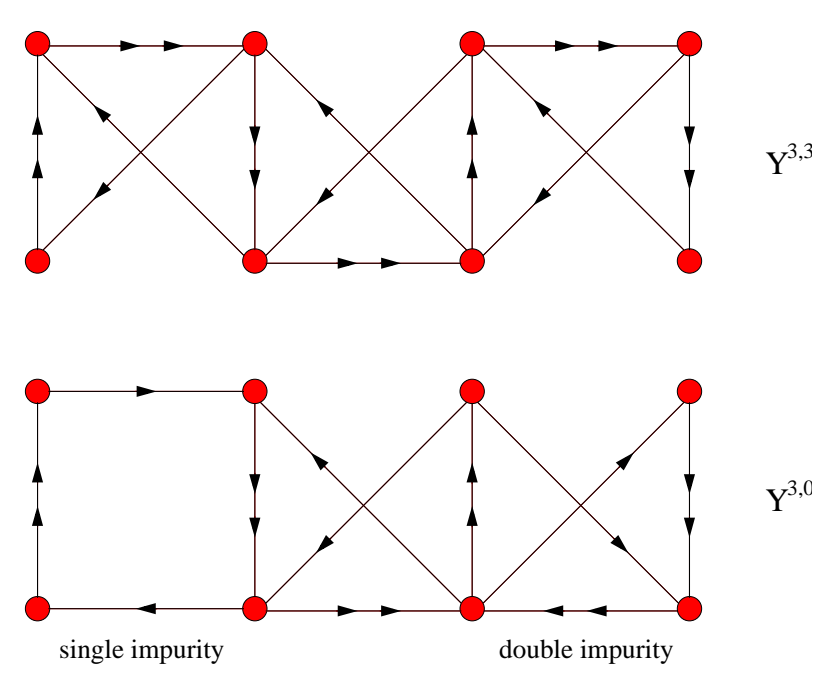

Figure 16: The quivers for $Y^{33}$ and $Y^{30}$ with one single (left block) and one double impurity (right block).

In these toric phases of the $Y^{p, q}$ theories, the only self-dual nodes are the ones at the ends of a single or double impurity. The blow-up procedure which produces the $X^{p, q}$ theories creates new self-dual nodes. More precicely, node $B$ in $Y^{p, q}$ Figure 0 is replaced by self-dual nodes $B_{1}$ and $B_{2}$ in $X^{p, q}$ Figure 8. Dualizing gauge groups that do not share any bifundamentals with these two nodes has exactly the same effect as in the case of $Y^{p, q}$. The impurities of $Y^{p, q}$ can be moved around the quiver, fusing into double impurities when they collide. As an example, we show the result of dualizing node 6 of $X^{3,1}$ below. The two impurities fuse into a double impurity exactly as they would in the absence of the blow up. Dualizing node 7 has an essentially identical effect.

On the other hand, dualizing nodes connected by an arrow to the new self-dual nodes leads to new theories, not always accounted for by the toric phase structure of $Y^{p, q}$. Again we will use $X^{3,1}$ as a showcase. Dualizing node 4 leads to the quiver shown in Figure 18. We have moved node 4 between nodes 1 and 2 to make clear the result of this dualization: The theory we get is the same as the one we would get by blowing up node 2 instead of 3, as mentioned in Section 3. Higgsing

\footnotetext{
${ }^{6}$ crenellating, adj.: having repeated square indentations like those in a battlement.
} 

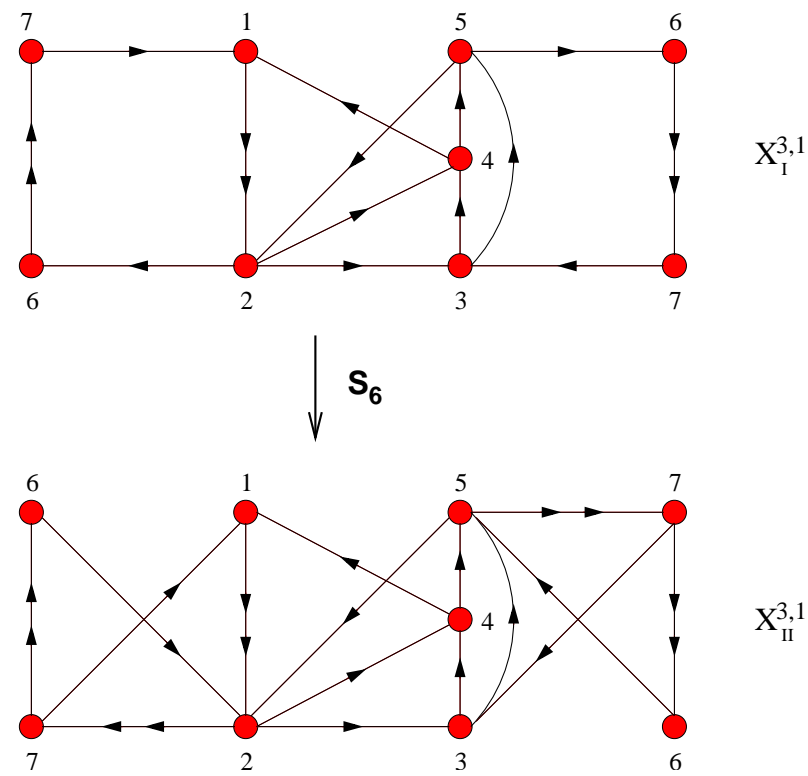

Figure 17: The notation $S_{6}$ means Seiberg duality on node 6. The resulting double impurity is located between nodes 1 and 6 .

$X_{14}$ in this quiver gives $Y^{3,1}$ with two single impurities, while Higgsing $X_{42}$ gives $Y^{3,0}$ with three single impurities. Dualizing 5 gives a theory completely equivalent to this one.

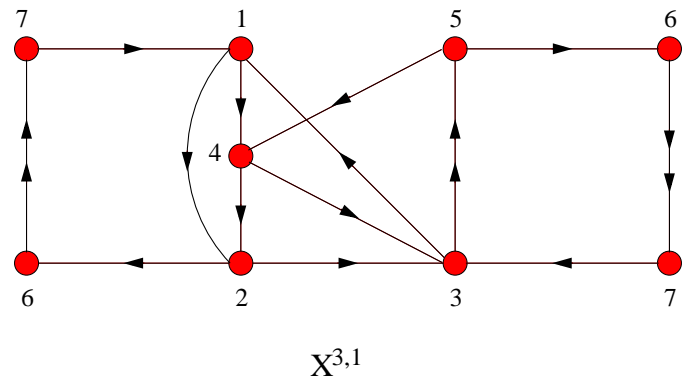

Figure 18: Seiberg duality on node 4 corresponds to blowing up a different node of $Y^{3,1}$.

The phases of $X^{3,1}$ we have described so far have either 15 or 19 fields. When we Higgs them down to $Y^{3,1}$ only the field that gets a vev disappears and we get the phases with with 14 and 18 fields respectively. Higgsing to $Y^{3,0}$ gives mass to two fields which are integrated out and removed from the massless spectrum together with the field that gets the expectation value, producing the toric phases with 12 and 16 fields. The difference in the number of fields simply comes from the difference in the distribution of impurities in $Y^{3,1}$. Whenever two single impurities fuse into a double impurity in $Y^{p, q}$, the number of fields goes up by four. In addition to these, there are also "intermediate" toric phases of $X^{3,1}$ that have 17 fields. These blow down to the phase of $Y^{3,1}$ that has 14 fields and to the phase of $Y^{3,0}$ with 16 fields. The details of the Higgsing are now reversed: 
two additional fields are integrated out when going to $Y^{3,1}$, but only the field with a vev disappears when we blow down to $Y^{3,0}$. For $X^{3,1}$ there are precisely two such phases, produced by dualizing nodes 1 or 3 . In Figure 19 we show the first of these two, and the phases of $Y^{p, q}$ to which it blows down.

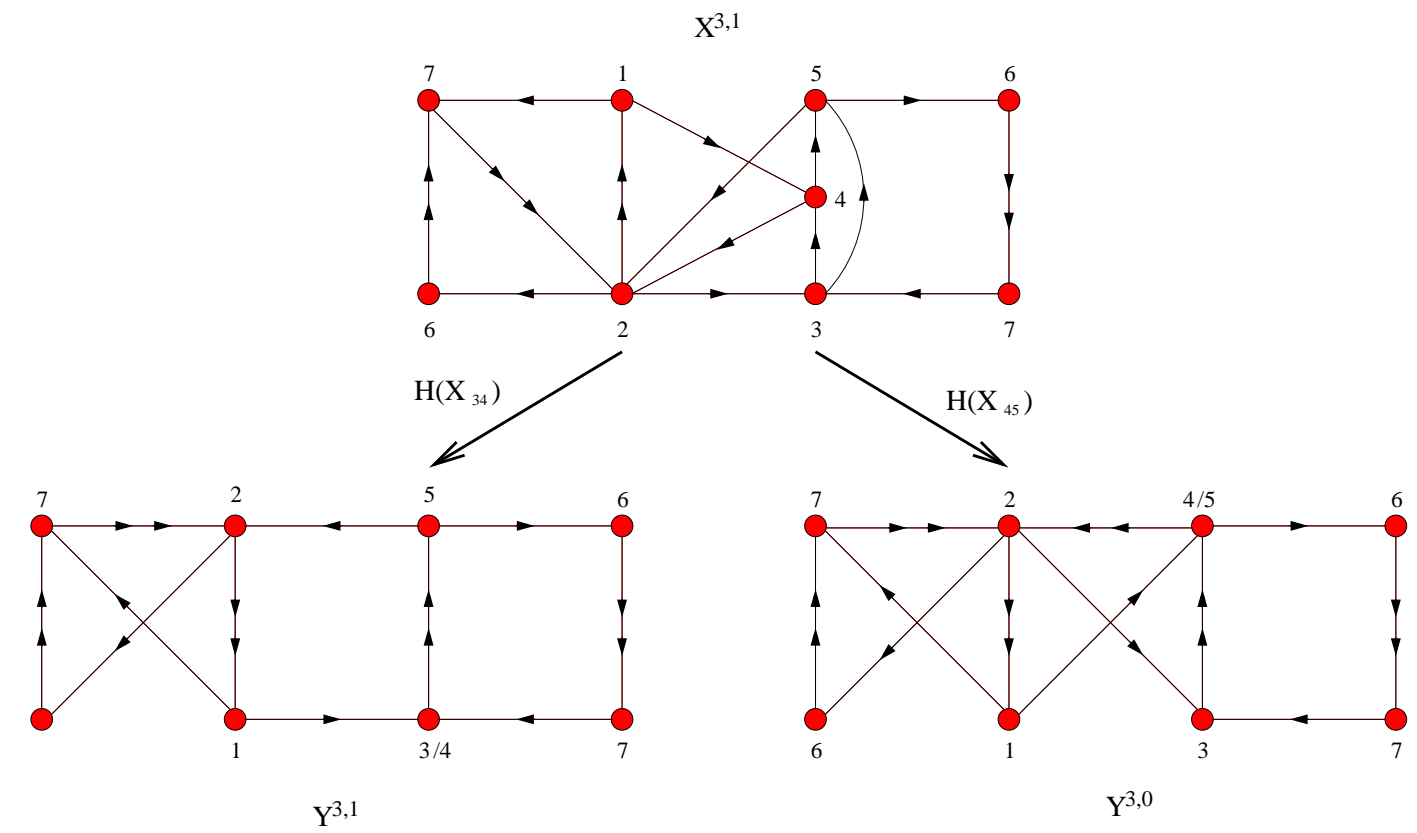

Figure 19: One of the two toric phases of $X^{3,1}$ with 17 fields. The notation $H\left(X_{i j}\right)$ means giving a vev to the scalar component of $X_{i j}$.

More generally, we expect the toric phases of all $X^{p, q}$ to fall in this pattern. Increasing $p$ does not change the story, because the blow up is localized on the quiver graph. The $Y^{p, q}$ theories have toric phases with $4 p+2 q+4 n_{2}$ fields where $n_{2}=0,1, \ldots\left[\frac{p-q}{2}\right]$ is the number of double impurities. For each of these models there will be toric phases of $X^{p, q}$ with one additional field that Higgs down to $Y^{p, q}$ and $Y^{p, q-1}$ like the examples in Figures 17,18. On top of these we have the "intermediate" phases with $4 p+2 q+4 n_{2}+3$ fields, where $n_{2}=0,1, \ldots\left[\frac{p-q}{2}\right]-1$. These also blow down to $Y^{p, q}$, $Y^{p, q-1}$ in the way described for the example in Figure 19. This is summarized in in Figure 20 for the case of $X^{3,1}$. It would be interesting to have a more general understanding of the Seiberg dual phases of $X^{p, q}$, including non-toric ones, since it may be easier to extract information about the infrared dynamics from some of these models than from others.

The toric phases of $Y^{p, q}$ can be easily enumerated. The problem is the same as counting the ways of coloring the $p$ vertices of a $p$-gon using three different colors (corresponding to a single impurity, a double impurity, or no impurity) modulo the action of the dihedral group $D_{p}$. This is a standard application of Pólya's enumeration theorem [37]. The cycle index of the dihedral group 


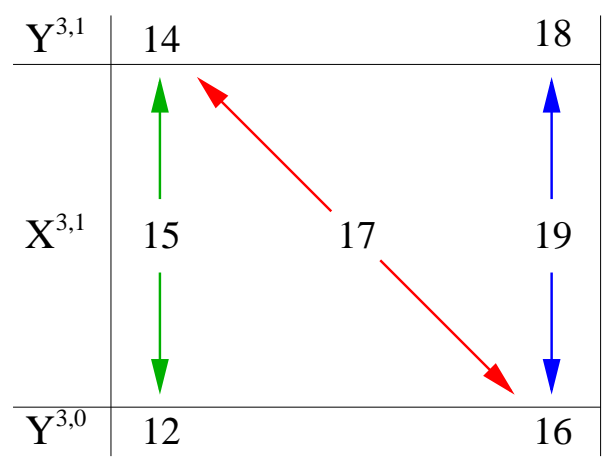

Figure 20: The number of fields for the toric phases of $X^{31}, Y^{31}, Y^{30}$ and the possible higgsings (shown with arrows). Note that several different toric phases can have the same number of fields.

is

$$
\begin{aligned}
\mathcal{Z}\left(D_{p}\right) & =\frac{1}{2} \mathcal{Z}\left(Z_{p}\right)+\frac{1}{2} x_{1} x_{2}^{(p-1) / 2} & & (p \text { odd }) \\
\mathcal{Z}\left(D_{p}\right) & =\frac{1}{2} \mathcal{Z}\left(Z_{p}\right)+\frac{1}{4}\left(x_{2}^{p / 2}+x_{1}^{2} x_{2}^{(p-2) / 2}\right) & & (p \text { even })
\end{aligned}
$$

where $\mathcal{Z}\left(Z_{p}\right)$ is the cycle index of the cyclic group of order $p$ :

$$
\mathcal{Z}\left(Z_{p}\right)=\frac{1}{p} \sum_{n \mid p} \phi(n) x_{n}^{p / n}
$$

and $\phi(n)$ is Euler's totient function. To implement the condition $n_{1}+2 n_{2}=p-q$ we assign weight 1 for no impurity, $\lambda$ to a single impurity and $\lambda^{2}$ to a double impurity. Then $x_{n}=1+\lambda^{n}+\lambda^{2 n}$, and

after plugging this into (5.1) the number of toric phases is given by the coefficient of $\lambda^{p-q}$ in the resulting polynomial. Because of the way the $X^{p, q}$ theories Higgs to $Y^{p, q}$ and $Y^{p, q-1}$, we expect the number of toric phases of $X^{p, q}$ to be essentially determined by (and in fact greater than) the number of toric phases of $Y^{p, q}$, but we shall leave a detailed counting to future work.

Finally, we note that when performing Seiberg duality one must alter the superpotential as well as the quiver. This is done by including cubic interactions of the form $M q \tilde{q}$, where $q$ and $\tilde{q}$ are the dual quarks and $M$ is a composite field in the original theory which maps to a singlet in the dual. One must be careful when applying this procedure to bifundamentals, since here the field $M$ is only a singlet under the dualized group but still transforms as a bifundamental in the dual theory. This procedure works as expected for the $X^{p, q}$ theories and produces the necessary $Y^{p, q}$ superpotentials upon giving a vev to the appropriate bifundamentals. For more details, we refer the reader to [38, 39].

\section{R-charges}

An important test of the AdS/CFT correspondence is that the dimensions of operators in the field theory match with the volumes of the different supersymmetric (calibrated) submanifolds D3- 
branes can wrap in the geometry [40, 41]. These dimensions, or equivalently R-charges, can be figured out in the gauge theory via $a$-maximization, and in the geometry via a volume calculation. These charges have also been computed via techniques from algebraic geometry [42, 43, 44, 45]. It is also possible to derive some of the R-charges purely from the toric data [30] ${ }^{7}$.

Let's briefly review the situation for $d P_{2}$, which will reveal an interesting aspect of the $X^{p, q}$ theories. The quiver for $d P_{2}$ is given in Figure 1. The R-charges for this theory are known [13, and given in Table 2. Since baryonic operators in the SCFT correspond to D3-branes wrapping 3 -cycles, we may associate to each bifundamental a 3 -manifold in the dual geometry. Since $d P_{2}$ has two 3 -cycles, we expect two baryonic $U(1)$ global symmetries. Similarly, we expect $d P_{2}$ to have a $U(1) \times U(1)$ isometry, which should translate to an additional $U(1)^{2}$ flavor symmetry. The baryonic $U(1)$ 's do not mix with the R-symmetry, as discussed in [12, 42]. Therefore there should be a twodimensional space of $U(1)$ symmetries which can potentially mix with the R-symmetry. Indeed, we note that we may assign a 2-dimensional basis of R-charges to the fields in $d P_{2}$ as given in Table 2. One can see a similar agreement for the $Y^{p, q}$ theories: With one non-baryonic $U(1)$ symmetry, one can reduce the $\mathrm{R}$-charges to a one-dimensional basis. This is what allowed the authors of [11] to compute the R-charges of these theories.

Equivalently, we can rephrase the above discussion as follows: Since the baryonic symmetries do not mix with the R-charges, it should be possible to do $a$-maximization over a two-dimensional space of parameters for $d P_{2}$ and over a one-dimensional space for $Y^{p, q}$. Naively, one might think from the field theory that $d P_{2}$ would require $a$-maximization over four parameters; this is what comes out of solving the linear equations given by the constraints of anomaly freedom at each node, and that the superpotential terms all have R-charge equal to two. However, we know that this is not the whole story; if one were able to pick the two flat directions properly, $a$-maximization could be done over only two parameters. This is easy to do once we know the right R-charges. From these, it is possible to work backwards and assign a good two-dimensional basis of charges as in Table 2. Doing a-maxmization over these two-dimensional basis, treating these charges as free parameters, yields the correct result.

One would naively think that just as the $d P_{1}$ results extended to general $Y^{p, q}$, we could extend the $d P_{2}$ results to general $X^{p, q}$. Since the number of baryonic $U(1)$ 's is always the number of external legs of the toric diagram minus three, there should always be two $U(1)_{B}$ symmetries in the $X^{p, q}$ theories that do not mix with the R-symmetry. Since we expect there to be naively four free parameters for the $X^{p, q}$ theories in general (we have verified this for e.g. $X^{3,1}$ although not for general $X^{p, q}$ ), there should be a remaining two-dimensional basis of $\mathrm{R}$-charges.

Unfortunately, however, we have not been able to reduce the R-charges of these theories to a two-dimensional basis. One difficulty is that since $a$-maximization for the $X^{p, q}$ theories must be done over four parameters (at least initially, since it is difficult to pick the flat directions in

\footnotetext{
${ }^{7}$ We thank James Sparks and Dario Martelli for alerting us to this work, and also for their comments, especially on this section of this paper.
} 


\begin{tabular}{|c|c|c|}
\hline Field & $R$ - charge & Linear Combination \\
\hline \hline$X_{52}$ & $\frac{3}{16}(19-3 \sqrt{33})$ & $\Sigma_{1}$ \\
\hline$X_{14}$ & $\frac{3}{16}(19-3 \sqrt{33})$ & $\Sigma_{1}$ \\
\hline$X_{53}$ & $\frac{1}{4}(9-\sqrt{33})$ & $\Sigma_{2}$ \\
\hline$X_{34}$ & $\frac{1}{4}(9-\sqrt{33})$ & $\Sigma_{2}$ \\
\hline$X_{42}$ & $\frac{1}{16}(-21+5 \sqrt{33})$ & $\Sigma_{2}-\Sigma_{1}$ \\
\hline$X_{51}$ & $\frac{1}{16}(-21+5 \sqrt{33})$ & $\Sigma_{2}-\Sigma_{1}$ \\
\hline$X_{23}^{1}$ & $\frac{1}{16}(-21+5 \sqrt{33})$ & $\Sigma_{2}-\Sigma_{1}$ \\
\hline$X_{31}^{1}$ & $\frac{1}{16}(-21+5 \sqrt{33})$ & $\Sigma_{2}-\Sigma_{1}$ \\
\hline$X_{23}^{2}$ & $\frac{1}{16}(17-\sqrt{33})$ & $\Sigma_{2}-\frac{1}{3} \Sigma_{1}$ \\
\hline$X_{31}^{2}$ & $\frac{1}{16}(17-\sqrt{33})$ & $\Sigma_{2}-\frac{1}{3} \Sigma_{1}$ \\
\hline$X_{45}$ & $\frac{1}{2}(-5+\sqrt{33})$ & $\Sigma_{2}-\frac{4}{3} \Sigma_{1}$ \\
\hline
\end{tabular}

Table 2: R-charges for $d P_{2}$. All R-charges can be expressed as a linear combination of two basis charges.

practice), it is very difficult to obtain exact numbers for $p>2$. We have, however, numerically computed the R-charges for $X^{3, q}$. Since the numbers are not particularly illuminating, we do not record the R-charges here. We do note that it appears not to be true that there is a two-dimensional basis of R-charges for general $X^{p, q}$ theories. One can easily check if, given three R-charges, there is any integer linear combination of them that equals another integer. To the precision allowed by Mathematica, we have not found any such linear combination of R-charges for $X^{3, q}$ for any $1 \leq q \leq 3$. Thus, it appears that our naive guess that there are only two $U(1)$ flavor symmetries in the Einstein-Sasaki manifold is incorrect. We note that the $X^{p, p}$ theory is special in that the quiver and superpotential have a $\mathbb{Z}_{2}$ symmetry which gives a nontrivial constraint on the R-charges; in these cases we may reduce the number of independent R-charges to three. Thus, we have a puzzle: Since the baryonic $U(1)$ 's should not mix with the R-symmetry, there should be relations between the R-charges. Our inability to find such relations may be a consequence of our numerical computation; since we cannot find the exact R-charges for the $X^{p, q}$, we can only check that things sum to zero up to a given numerical precision. However, it is possible that there is a deeper issue here as well.

Finally, we note that there are some $X^{p, q}$ where we can actually find the R-charges exactly. We have done the calculation for $X^{2,2}$ and $X^{3,3}$, and found that in these cases the R-charges are not quadratic irrational, but instead the roots of quartic polynomials. These are the first examples of theories whose R-charges are not quadratic irrationals. This is not in contradiction to the prediction from $a$-maximization, since the charges are still algebraic numbers. Since $a$ is a cubic function over many variables, derivatives of $a$ will be quadratic functions over many variables. In general the 
solutions of these equations will not be quadratic irrational, although they have been for every case studied so far. Although we do not record the exact values of all the R-charges for $X^{2,2}$ and $X^{3,3}$ here, we do note for the sake of reference two R-charges. For $X^{3,3}$ (shown in Figure 10), the R-charge of the bifundamental $X_{56}$ is given by a root of the polynomial $27 x^{4}-198 x^{3}-180 x^{2}+650 x-250$. For $X^{2,2}$ (also known as Pseudo-del Pezzo 2), the R-charge of one of the fields ( $X_{53}$ in Figure 6 of [46]) is given by a root of $9 x^{4}-78 x^{3}+112 x^{2}+16 x+32$.

\section{Acknowledgements}

We would like to thank Alan Dunn, Sebastian Franco, Dan Freedman, Barak Kol, Dario Martelli, and James Sparks for awesome discussions. BW would like to thank Nick Jones and his amusing accent for last-minute TeX help, and acknowledge the hospitality of Brook Williams's apartment, where the final stages of this work were completed. This work was supported in part by the CTP and LNS of MIT and the U.S. Department of Energy under cooperative research agreement \# DE-FC02- 94ER40818, National Science Foundation Grant PHY-00-96515, and by the BSF American-Israeli Bi-National Science Foundation. A. H. is also indebted to a DOE OJI Award.

\section{References}

[1] M. R. Douglas and G. W. Moore, "D-branes, Quivers, and ALE Instantons," arXiv:hep-th/9603167.

[2] J. M. Maldacena, "The large N limit of superconformal field theories and supergravity," Adv. Theor. Math. Phys. 2, 231 (1998) [Int. J. Theor. Phys. 38, 1113 (1999)] arXiv:hep-th/9711200.

[3] S. S. Gubser, I. R. Klebanov and A. M. Polyakov, "Gauge theory correlators from non-critical string theory," Phys. Lett. B 428 (1998) 105 [arXiv:hep-th/9802109].

[4] E. Witten, "Anti-de Sitter space and holography," Adv. Theor. Math. Phys. 2 (1998) 253 [arXiv:hep-th/9802150].

[5] D. R. Morrison and M. R. Plesser, "Non-spherical horizons. I," Adv. Theor. Math. Phys. 3, 1 (1999) [arXiv:hep-th/9810201].

[6] A. Kehagias, "New type IIB vacua and their F-theory interpretation," Phys. Lett. B 435, 337 (1998) [arXiv:hep-th/9805131].

[7] J. P. Gauntlett, D. Martelli, J. Sparks and D. Waldram, "Sasaki-Einstein metrics on S(2) x S(3)," arXiv:hep-th/0403002.

[8] J. P. Gauntlett, D. Martelli, J. Sparks and D. Waldram, "Supersymmetric AdS(5) solutions of M-theory," Class. Quant. Grav. 21, 4335 (2004) [arXiv:hep-th/0402153].

[9] D. Martelli and J. Sparks, "Toric geometry, Sasaki-Einstein manifolds and a new infinite class of AdS/CFT duals," arXiv:hep-th/0411238.

[10] B. Feng, A. Hanany and Y. H. He, "D-brane gauge theories from toric singularities and toric duality," Nucl. Phys. B 595, 165 (2001) [arXiv:hep-th/0003085]. 
[11] S. Benvenuti, S. Franco, A. Hanany, D. Martelli and J. Sparks, "An infinite family of superconformal quiver gauge theories with Sasaki-Einstein duals," arXiv:hep-th/0411264.

[12] K. Intriligator and B. Wecht, "The exact superconformal R-symmetry maximizes a," Nucl. Phys. B 667, 183 (2003) [arXiv:hep-th/0304128].

[13] M. Bertolini, F. Bigazzi and A. L. Cotrone, "New checks and subtleties for AdS/CFT and a-maximization," JHEP 0412 (2004) 024 [arXiv:hep-th/0411249].

[14] S. Franco, A. Hanany and A. M. Uranga, "Multi-flux warped throats and cascading gauge theories," arXiv:hep-th/0502113.

[15] A. Bergman, "Undoing orbifold quivers," arXiv:hep-th/0502105.

[16] S. Benvenuti and A. Hanany, "Conformal manifolds for the conifold and other toric field theories," arXiv:hep-th/0502043.

[17] S. S. Pal, "A new Ricci flat geometry," arXiv:hep-th/0501012.

[18] S. Benvenuti, A. Hanany and P. Kazakopoulos, "The toric phases of the Y(p,q) quivers," arXiv:hep-th/0412279.

[19] S. Franco, Y. H. He, C. Herzog and J. Walcher, "Chaotic cascades for D-branes on singularities," arXiv:hep-th/0412207.

[20] C. P. Herzog, Q. J. Ejaz and I. R. Klebanov, "Cascading RG flows from new Sasaki-Einstein manifolds," JHEP 0502, 009 (2005) [arXiv:hep-th/0412193].

[21] I. R. Klebanov and E. Witten, "Superconformal field theory on threebranes at a Calabi-Yau singularity," Nucl. Phys. B 536, 199 (1998) [arXiv:hep-th/9807080].

[22] B. Feng, A. Hanany and Y. H. He, "Phase structure of D-brane gauge theories and toric duality," JHEP 0108, 040 (2001) [arXiv:hep-th/0104259].

[23] N. C. Leung and C. Vafa, "Branes and toric geometry," Adv. Theor. Math. Phys. 2, 91 (1998) [arXiv:hep-th/9711013].

[24] W. Fulton, "Introduction to Toric Varieties," Princeton University Press, 1993.

[25] O. Aharony, A. Hanany and B. Kol, "Webs of (p,q) 5-branes, five dimensional field theories and grid diagrams," JHEP 9801, 002 (1998) [arXiv:hep-th/9710116].

[26] O. Aharony and A. Hanany, "Branes, superpotentials and superconformal fixed points," Nucl. Phys. B 504, 239 (1997) [arXiv:hep-th/9704170].

[27] A. Iqbal and V. S. Kaplunovsky, "Quantum deconstruction of a 5D SYM and its moduli space," JHEP 0405, 013 (2004) [arXiv:hep-th/0212098].

[28] A. Iqbal and A. K. Kashani-Poor, "SU(N) geometries and topological string amplitudes," arXiv:hep-th/0306032.

[29] M. R. Douglas, S. Katz and C. Vafa, "Small instantons, del Pezzo surfaces and type I' theory," Nucl. Phys. B 497, 155 (1997) [arXiv:hep-th/9609071]. 
[30] D. Martelli, J. Sparks, S. T. Yau, arXiv:hep-th/0503186.

[31] A. Hanany and K. D. Kennaway, "Dimer models and toric diagrams," arXiv:hep-th/0503149.

[32] S. Franco, A. Hanany, K. Kennaway, D. Vegh, B. Wecht, to appear.

[33] B. Feng, S. Franco, A. Hanany and Y. H. He, "Unhiggsing the del Pezzo," JHEP 0308, 058 (2003) [arXiv:hep-th/0209228].

[34] S. Franco and A. Hanany, "Geometric dualities in 4d field theories and their 5d interpretation," JHEP 0304, 043 (2003) [arXiv:hep-th/0207006].

[35] K. A. Intriligator, D. R. Morrison and N. Seiberg, "Five-dimensional supersymmetric gauge theories and degenerations of Calabi-Yau spaces," Nucl. Phys. B 497, 56 (1997) [arXiv:hep-th/9702198].

[36] N. Seiberg, "Electric - magnetic duality in supersymmetric nonAbelian gauge theories," Nucl. Phys. B 435, 129 (1995) [arXiv:hep-th/9411149].

[37] G. Pólya and R. C. Read, "Combinatorial enumeration of groups, graphs, and chemical compounds," Pólya's paper translated from German by Dorothee Aeppli, Springer-Verlag, New York, 1987.

[38] C. E. Beasley and M. R. Plesser, "Toric duality is Seiberg duality," JHEP 0112, 001 (2001) [arXiv:hep-th/0109053].

[39] B. Feng, A. Hanany, Y. H. He and A. M. Uranga, "Toric duality as Seiberg duality and brane diamonds," JHEP 0112, 035 (2001) [arXiv:hep-th/0109063].

[40] E. Witten, "Baryons and branes in anti de Sitter space," JHEP 9807, 006 (1998) [arXiv:hep-th/9805112].

[41] D. Berenstein, C. P. Herzog and I. R. Klebanov, "Baryon spectra and AdS/CFT correspondence," JHEP 0206, 047 (2002) [arXiv:hep-th/0202150].

[42] K. Intriligator and B. Wecht, "Baryon charges in 4D superconformal field theories and their AdS duals," Commun. Math. Phys. 245, 407 (2004) [arXiv:hep-th/0305046].

[43] C. P. Herzog, "Exceptional collections and del Pezzo gauge theories," JHEP 0404, 069 (2004) [arXiv:hep-th/0310262].

[44] C. P. Herzog and J. Walcher, "Dibaryons from exceptional collections," JHEP 0309, 060 (2003) [arXiv:hep-th/0306298].

[45] C. P. Herzog and J. McKernan, "Dibaryon spectroscopy," JHEP 0308, 054 (2003) [arXiv:hep-th/0305048].

[46] B. Feng, Y. H. He and F. Lam, "On correspondences between toric singularities and (p,q)-webs," Nucl. Phys. B 701, 334 (2004) [arXiv:hep-th/0403133]. 